University of Massachusetts Amherst

ScholarWorks@UMass Amherst

Doctoral Dissertations 1896 - February 2014

$1-1-1991$

\title{
The effect of the assumed boundary in the solving of the nine-dot problem on a sample of Chinese and American students 6-18 years old.
}

Jie Li

University of Massachusetts Amherst

Follow this and additional works at: https://scholarworks.umass.edu/dissertations_1

\section{Recommended Citation}

$\mathrm{Li}$, Jie, "The effect of the assumed boundary in the solving of the nine-dot problem on a sample of Chinese and American students 6-18 years old." (1991). Doctoral Dissertations 1896 - February 2014. 4780.

https://scholarworks.umass.edu/dissertations_1/4780

This Open Access Dissertation is brought to you for free and open access by ScholarWorks@UMass Amherst. It has been accepted for inclusion in Doctoral Dissertations 1896 - February 2014 by an authorized administrator of ScholarWorks@UMass Amherst. For more information, please contact scholarworks@library.umass.edu. 

UMASS/AMHERST 


\title{
THE EFFECT OF THE ASSUMED BOUNDARY IN THE SOLVING OF THE NINE-DOT PROBLEM ON A SAMPLE OF CHINESE AND AMERICAN STUDENTS 6-18 YEARS OLD
}

A Dissertation Presented

BY

\section{CHIEH LI}

\author{
Submitted to the Graduate School of the \\ University of Massachusetts in partial fulfilment \\ of the requirements for the degree of \\ DOCTOR OF EDUCATION \\ February, 1991
}

Education 
THE EFFECT OF THE ASSUMED BOUNDARY IN THE SOLVING OF THE NINE-DOT PROBLEM ON A SAMPLE OF CHINESE AND AMERICAN STUDENTS 6-18 YEARS OLD

A Dissertation Presented

By

CHIEF LI

Approved as to style and content by:

Ina Vazquy nut ere

Ina Vazquez Nuttall, Chair

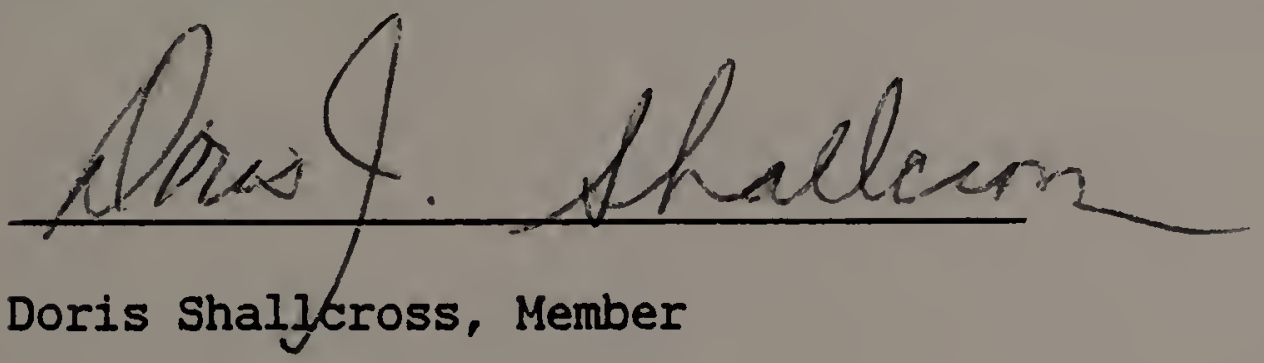

Mani Dash

Marvin Daehler, Member

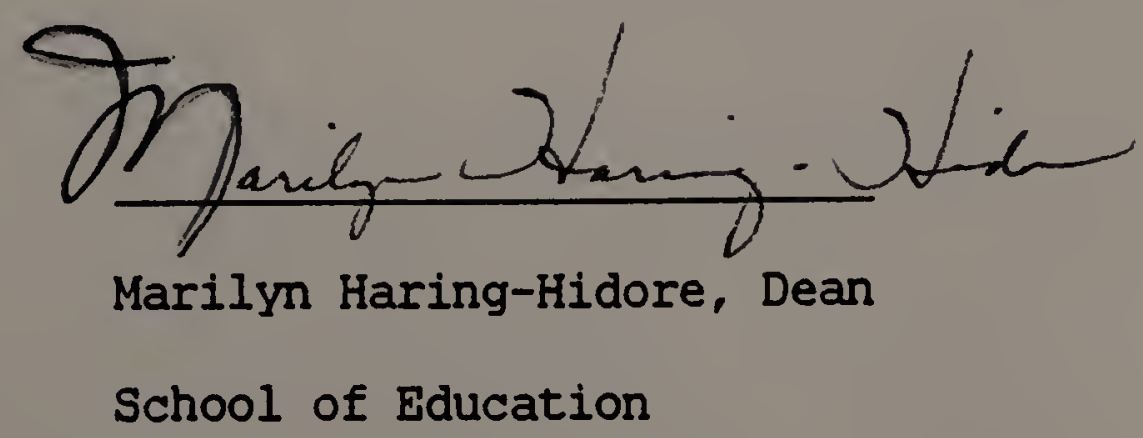




\section{ACKNOWLEDGEMENTS}

It is difficult to complete a study like this without the support and assistance of many people. First and foremost, I am very grateful to all members of my dissertation committee. I can never thank enough Dr. Ena V. Nuttall, my committee chair, for her valuable advice from her rich experience in cross-cultural work, her deep care and love and her forever availability. My committee member Dr. Doris Shallcross, who is devoted to creative education, has introduced me to colleagues and resources of creativity studies and aroused my interest in studies of the effect of assumed boundaries. Her support and encouragement helped me in developing and carrying out this project. I owe so much to Dr. Marvin Daehler, my committee member, for his stimulating questions, his constructive criticism based on his incredible knowledge and experience in developmental studies, and, above all, for his great effort going through this whole thesis word by word. He literally edited this paper.

This study would have been impossible but for the enthusiasm and participation of students, teachers and principals of elementary and high schools in Beijing, the People's Republic of China, and in Massachusetts, U.S.A. Heart-felt thanks to their valuable time and contribution.

I also owe a debt to my parents and my husband who are a source of persistent encouragement and volunteer assistance. 


\begin{abstract}
Finally, this study has been supported by the Creative
Education Foundation and School of Education of University of

\section{Massachusetts.

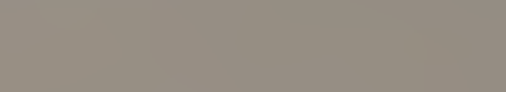

(n)

Finally, this study has been supported by the Creative
Education Foundation and School of Education of University of
\end{abstract}

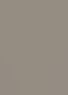

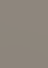


ABSTRACT

THE EFEECT OF THE ASSUMED BOUNDARY IN THE SOLVING OF

THE NINE-DOT PROBLEM ON A SAMPLE OF CHINESE AND AMERICAN

STUDENTS 6-18 YEARS OLD

FEBRUARY, 1991

CHIEH LI, B.A., BEIJING TEACHERS COLIEGE

M.A., BEIJING TEACHERS COLLEGE

Ed.D. , UNIVERSITY OF MASSACHUSETTS

Directed by: Professor Ena V. Nuttall

The nature of the difficulty of nine-dot problem solving has been controversial. A commonly accepted explanation is that problem solvers often fixate on the square shape of the dot pattern and confine their lines to the square area. This study was designed to test how the phenomenon of the assumed boundary is affected by culture, age and sex.

The study used the nine-dot problem as the task and age, culture and sex as independent variables. One hundred and sixty mainstreamed Chinese and mainstreamed American participants from four age groups: $6-7,10-11,15-16,17-18$, half of whom were males and half of whom were females, participated. The data examined to analyze participants' problem solving processes were: 1) the number of solution attempts, 2) the time spent before and after extending the boundary formed by the dots, and 3 ) the total time spent in solving the problem, or, in working on the problem, if participants gave up before finding the solution. 
The results of the study revealed a significant cultural and age effect in the number of successful solvers and the time spent on the problem. Although the overall sex difference was not significant an interaction between culture and sex was found. American girls spent less time on solving the problem than boys while Chinese girls spent more time than boys to solve the problem. Additional findings of this study were 1) a new version of solution to the nine-dot problem and, 2) evidence that Chinese children who had taken thinking courses could solve the nine-dot problem more effectively than children who had not. The implications and limitations of the study were discussed, and recommendations for further research were made. 
TABLE OF CONTENTS

Page

ACKNOWLEDGMENT $\ldots \ldots \ldots \ldots \ldots \ldots \ldots \ldots \ldots \ldots \ldots \ldots \ldots \ldots$

ABSTRACT $\ldots \ldots \ldots \ldots \ldots \ldots \ldots \ldots \ldots \ldots \ldots \ldots \ldots \ldots \ldots \ldots \ldots \ldots$

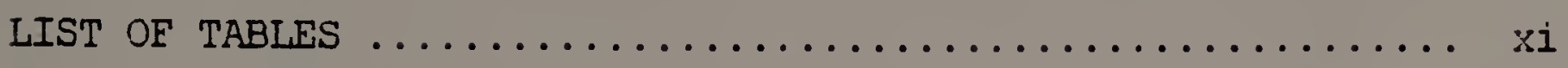

IIST OF FIGURES $\ldots \ldots \ldots \ldots \ldots \ldots \ldots \ldots \ldots \ldots \ldots \ldots \ldots \ldots \ldots \ldots \ldots$

Chapter

1 INTRODUCTION $\ldots \ldots \ldots \ldots \ldots \ldots \ldots \ldots \ldots \ldots \ldots \ldots \ldots \ldots$

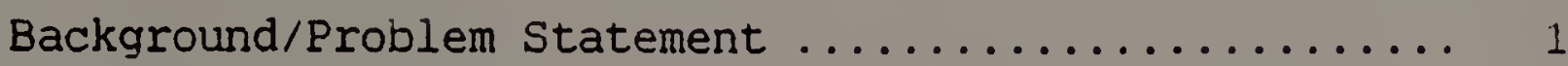

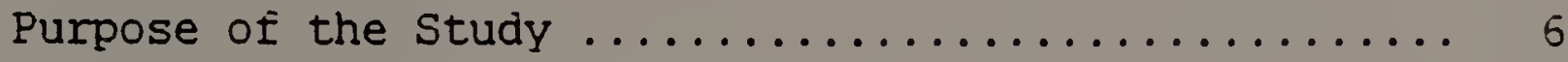

Organization of the Dissertation $\ldots \ldots \ldots \ldots \ldots \ldots \ldots \ldots 6$

2 LITERATURE REVIEW $\ldots \ldots \ldots \ldots \ldots \ldots \ldots \ldots \ldots \ldots \ldots$

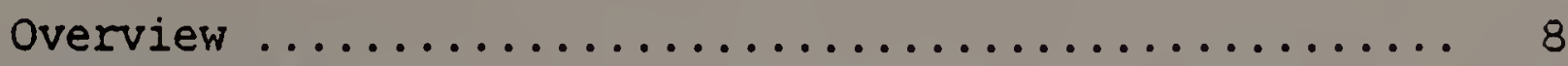

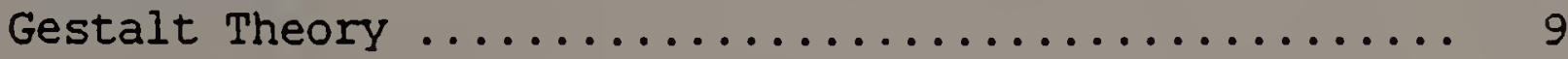

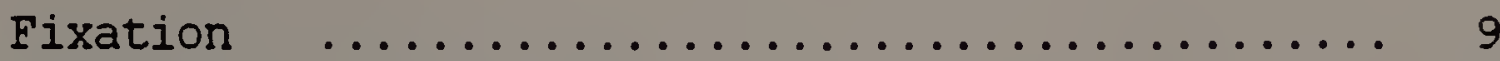

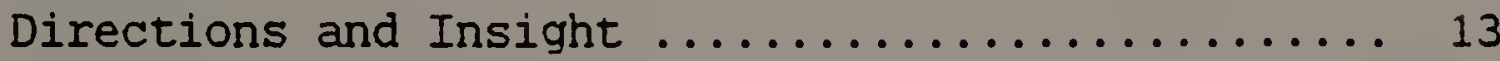

Retrieval/Hypothesis Model ................. 13

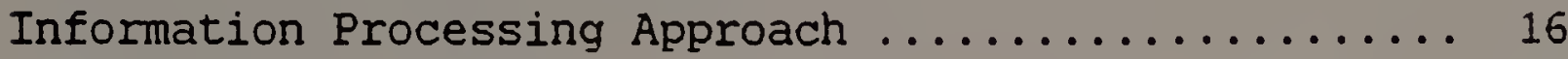

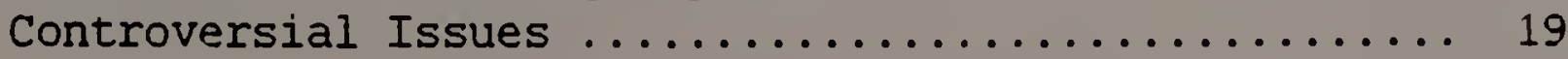

Are "Fixation" and "Insight" Useful Concepts? .... 19

What Is the Nature of the Difficulty of the

Nine-Dot Problem? ................. 22

Summary of Literature Review $\ldots \ldots \ldots \ldots \ldots \ldots \ldots \ldots$

3 METHODOLOGY $\ldots \ldots \ldots \ldots \ldots \ldots \ldots \ldots \ldots \ldots \ldots \ldots \ldots \ldots \ldots \ldots \ldots$

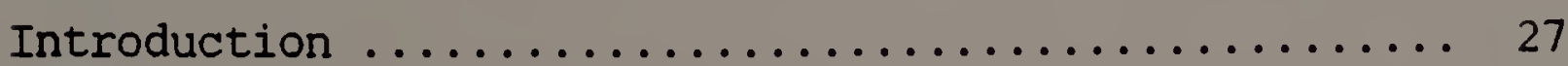

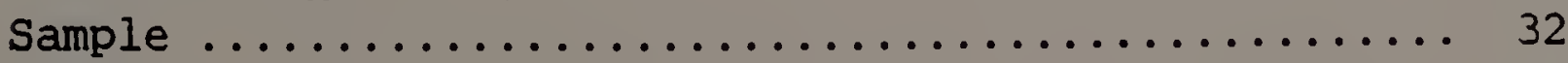

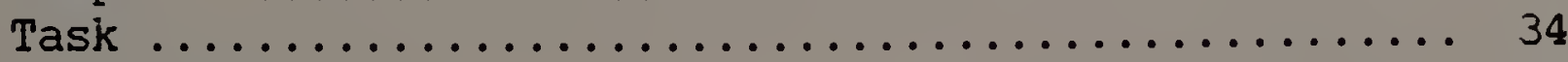

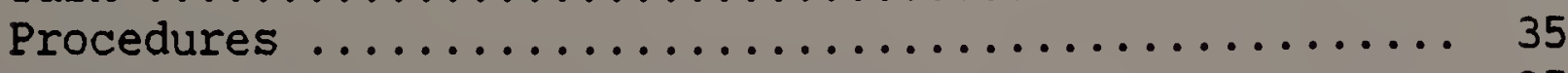

Research Design and Data Analysis ............. 35

4 RESULTS $\ldots \ldots \ldots \ldots \ldots \ldots \ldots \ldots \ldots \ldots \ldots \ldots \ldots \ldots \ldots \ldots$

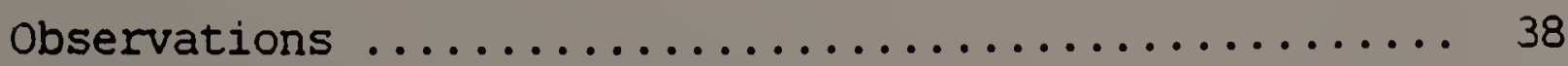

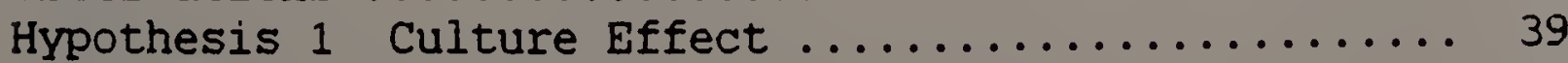

Hypothesis 2 Age Effect .................... 44 
Hypothesis 3 Sex Effect ................. 49

Hypothesis 4 Interaction between Culture and Age .... 49

Hypothesis 5 Interaction between Culture and Sex .... 53

Additional Findings ................... 56

5 DISCUSSION $\ldots \ldots \ldots \ldots \ldots \ldots \ldots \ldots \ldots \ldots \ldots \ldots \ldots \ldots \ldots \ldots \ldots \ldots$

Hypothesis 1 Culture Effect ................ 62

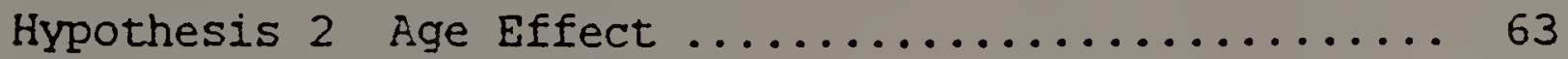

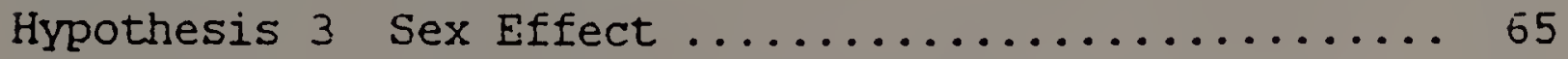

Hypothesis 4 Interaction between Culture and Age ... 66

Hypothesis 5 Interaction between Culture and Sex .... 67

Additional Findings .................... 68

The Nature of the Difficulty of the Nine-dot

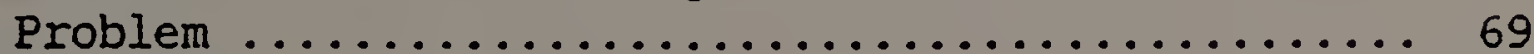

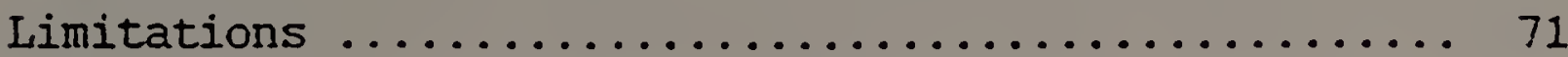

Recommendations for Further Research ........... 72

\section{APPENDICES}

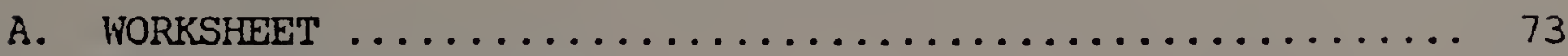

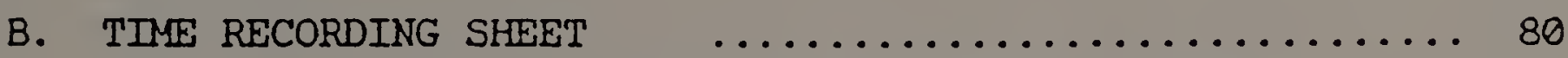

C. GRAPHS OF NINE-DOT PROBLEM AND ELEVEN-DOT PROBLEM $\ldots \ldots \ldots .81$

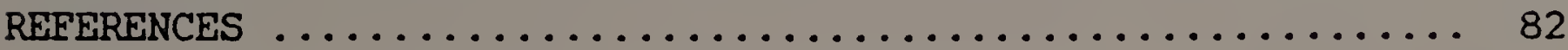




\section{LIST OF TABLES}

Table

3.1 A Summary of the Design for the Analysis

Page

of Variance ....................... 37

4.1 Nultivariate Tests of Significance

-- Time and Trials to Solve the Problem ...... 42

4.2 Multivariate Tests of Significance

-- Time and Trials to Break the Boundary ......

4.3 Multivariate Tests of Significance

-- Time and Trials by Unsuccessful Solvers

on the Problem ...................... 43

4.4 Summary of Univariate $F$ Tests on Cultural

Effect .......................... 43

4.5 Number of Subjects Producing Lines Extending beyond the Boundary and Solving the Problem ... 45

4.6 The Average Time Spent by Each Age Group

to Break the Boundary, to Solve the Problem,

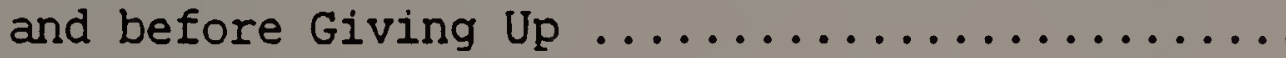

4.7 The Average Trials Made by Each Age Group

to Break the Boundary, to Solve the Problem

and before Giving Up ................ 47

4.8 Summary of Univariate $F$ Tests on Age Effect ..... 48

4.9 Summary of Univariate $F$ Tests on Interaction between Culture and Age $\ldots \ldots \ldots \ldots \ldots \ldots \ldots \ldots, 51$

4.10 Summary of Contrasts between Means of Age Groups in G-time, G-trial, B-time,

B-trial, S-time and S-trial

4.11 Percentage of the Boundary-Breakers Who Solved the Problem ............... 53

4.12 Average Time Spent by Each Sex Group to Solve the Problem .................. 54

4.13 Summary of Univariate $F$ Tests on Interaction between Culture and Sex $\ldots \ldots \ldots \ldots \ldots \ldots \ldots \ldots, 55$

4.14 Number of Boundary-Breakers, Problem-Solvers and Give-Ups within Twenty Trials ......... 57

4.15 Percentage of Students Who Drew the Shape of the Solution within the Boundary 


\section{LIST OF FIGURES}

\section{Figure}

4.1 Frequency Distribution of Trials on the

\section{Page}

Problem by Unsuccessful Solvers ........... 41

4.2 Triangle-Shape Solution within the Boundary ..... 59

4.3 The New Version of the Solution $\ldots \ldots \ldots \ldots \ldots$ 
CHAPTER 1

INTRODUCTION

\section{Background/Problem Statement}

The effect of an assumed boundary in problem solving was described in Edward de Bono's Lateral Thinking: Creativity Step by Step in the early 1970s (de Bono, 1973). He pointed out, "in order to live at all, one must be making assumptions all the time. Yet each of these assumptions is a cliche pattern which may be restructured to make better use of available information. In addition the restructuring of more complex patterns may prove impossible unless one breaks through some assumed boundary." (p.103). He used the nine-dot problem to illustrate his point. The problem is to connect all nine dots with four straight lines without retracing and without lifting the pencil from the paper (see APPENDIX A) Doris Shallcross also used the nine-dot problem to illustrate the importance of breaking the assumed boundary in her book Teaching Creative Behavior (1981).

The nine-dot problem has been referred to frequently in studies of the effect of assumed boundaries because of its stimulus pattern. First, the spatial organization of the nine dots as presented in the problem may hinder the achievement of the solution. The nine dots form a square but the solution requires the lines to move outside of the square. Second, the directions are not specified beyond the generally stated 
requirement "to solve the problem". It is not clearly stated a) whether the lines may be extended beyond the perimeter formed by the dots, b) whether one dot may be connected by two or three lines, c) whether the lines may cross each other, and d) whether the lines may intersect at points apart from a dot. Thus problem solvers are left to make assumptions about all these conditions.

In everyday life, many problems are not well defined; we have to make assumptions all the time. Sometimes the appearance of a problem is as misleading as the square in the nine-dot problem. Understanding the solution to the nine-dot problem may parallel creative problem solving in real life. For this reason, the problem is worth studying.

Western researchers (e.g., Maier, 1930; Scheerer, 1963) have found that people either take a long time to solve the nine-dot problem or fail to solve it. A popular explanation for the difficulty of the problem is that problem solvers often fixate on the square shape of the dot pattern and confine their lines to the square area (Scheerer, 1963). But the solution to the problem requires that lines go outside the square. Thus the problem becomes difficult because participants assume that lines must stay within the dots.

According to Piaget's (1970) theory of intellectual development, intelligence in the sensorimotor stage (0-2 years) is largely based on perceptual experience. At this stage, a child can not understand what the nine-dot problem means. But in the preoperational stage (2-7 years), language develops and thought 
becomes a symbolic process. Nevertheless, thinking is still perception bound. By the age of six or seven, most children may understand the question posed in the nine-dot problem but egocentrism, centration and lack of reversibility of thought may prevent children from derıving a solution which extends beyond the perceptual boundary formed by the dots. In the concrete operational stage $(7-11)$, thought becomes reversible, conservation becomes operative and ability to solve concrete problems develops. Logical operations develop although thinking remains largely experience based. Since the nine-dot problem is visual, it is possible for children during the late concrete operational stage to solve the problem. In the formal operational stage (12-adulthood), formulation and testing of hypotheses, abstract thought, deductive and hypothetio-deductive reasoning develop. Thought is no longer perception bound. At this stage, one is developmentally ready for the nine-dot problem. In short, cognitive development gradually equips people for the necessary mental ability to solve the nine-dot problem.

In addition to developmental factors. cultural background may play an important role in problem solving. Some linguists (Sapir, 1929; Whorf, 1956) argue that our view of the world depends on the particular language we have learned. Our perception of reality is affected by the words and the grammatical rules of our language which are acquired via our culture. If something is important to a people, their language will often contain many words to describe it. For instance, kinship is important to the 
Chinese people so that they have many words to describe different in-law relationships. Those words do not have counterparts in English. On the other hand, "privacy", a very important concept to Americans, does not have a linguistic equivalent in the rich Chinese vocabulary. Rules of grammar also enable us to see some things in certain ways and prevent us from seeing other things at all. For example, the tense of the English language reflects and reinforces the value of time for English speaking people. The four tones of Mandarin (a dialect of Chinese, standard Chinese) words make speakers of Chinese sensitive to tone change. In different cultures children are taught to pay attention to certain sounds and to ignore others (Hall, 1966a, 1966b). Once learned, these perceptual patterns tend to remain with people. It is not unreasonable to propose that these linguistic variations and their consequences on perception or attention have different effects on people's problem solving.

Three major dimensions of culture--the cognitive, the material, and the normative dimension--may also contribute to shaping ways of perceiving and thinking. The most important aspect of the cognitive dimension of culture is beliefs-ideas about what is thought to be true (Shepard, 1981). Material culture consists of the concrete, tangible objects within a culture. Objects of material culture have no meaning or use apart from the meanings and definitions given them by a group. The normative dimension of culture consists of ideas about what is normal behavior, i.e., what is expected of a member of a group. 
The most important aspects of the normative dimension are norms, sanctions, and values. Thus, people from different cultures may perceive the nine-dot problem differently and/or approach the problem differently. Therefore, cultural differences in the performance of the problem may be inevitable.

The universality of the existence of assumed perceptual boundaries may be questioned since the formation of perceptual and thinking patterns must be affected by cultural background and cognitive developmental levels. A comprehensive search of the available international literature did not yield any crosscultural or developmental studies on the effect of assumed boundaries on problem solving. Thus, in 1984, Li carried out pilot work to provide evidence for the effect of assumed boundaries on the solution of the nine-dot problem in a regular elementary school in Beijing, China. Thirty-two fifth graders, 10-11 years of age, participated. The task was given to the children as a group in the classroom. The time children spent trying to solve the problem, both before and after extending the square formed by the nine dots, was scored as well as the number of correct solutions. Children's worksheets of their problem solving were also examined. The results revealed that, at first, all children tried to solve the problem within the square space formed by the dots. The participants spent about 30 minutes on average before extending the boundary, but only 13 minutes on average after extending the boundary to find the solution. Eighty-eight percent of the children solved the problem. 
The course of problem solving was exactly as Shallcross described (1981, p.63). The results of this pilot work indicated that extending the assumed boundary was crucial in nine-dot problem solving for Chinese children 10-11 years old (the later years of concrete operations). However, additional empirical studies are clearly needed to expand the range of subjects included in the task. The current study was designed to continue to explore developmental and ethnic differences in work on the nine-dot problem solution.

\section{Purpose of the Study}

The purpose of this study was to provide evidence for the universality of the effect of the assumed boundary in the nine-dot problem by testing children of two different ethnic backgrounds and four developmental levels. Breaking the assumed boundaries or limits was hypothesized to be difficult for all people regardless of culture, age and sex. Mastering the strategy for challenging such an assumption should speed up problem solving processes associated with nine-dot problem.

\section{Organization of the Dissertation}

This dissertation consists of five chapters. This first chapter covered the background of the study, the problem statement, and the purpose of the study. In Chapter Two, an overview of research on the nine-dot problem is presented. Gestalt theory, a retrieval/hypothesis model, and an information processing approach are reviewed to highlight controversial issues over the nature of the difficulty of the nine-dot problem 
solving task. In Chapter Three, the methodology of the present study is presented. This chapter summarizes the specific hypotheses to be tested and describes the subject sample, the task, the procedures, the research design and method of data analysis. In Chapter Four, the results of this study are reported. In Chapter Five, discussion and conclusions about the hypotheses are dram, and implications of the findings and recommendations for further studies are made. 


\section{CHAPTER 2 \\ LITERATURE REVIEW}

\section{Overview}

Most cross-cultural studies of creativity have focused on measuring cognitive abilities such as ideational fluency, flexibility and originality (Rimm \& Davis, 1980; Torrance \& Sato. 1979; Lundsteen, 1980; Rabinsky \& Kumar, 1979). Some research has addressed the production of images (Khatena \& Zetenyi, 1983) and some the impact of cultural values on creativity in children (Hernandez, 1986). No studies, however, have addressed the extent to which assumed boundaries are observed in different cultures and at different developmental levels. The available literature that deals with the effect of assumed boundaries is found in research on insight problems.

Although the Gestalt psychologist Maier (1930) discussed the nine-dot problem and emphasized the effects of the square shape of the dots on problem difficulty as early as the 1930s, he did not provide any empirical data on how difficult the problem was. Scheerer (1963) also did not report empirical data on the nine-dot problem in his thorough review article of problem solving. In fact only four empirical studies (Maier \& Casselman, 1970; Burnham \& Davis, 1969; Weisberg \& Alba, 1981; Lung \& Dominowski, 1985) on the nine-dot problem could be found. All four studies used college students as subjects. No reports on 
developmental or cross-cultural studies of the nine-dot problem have been found. The issue of the universality of the effect of the assumed boundary was not addressed in any of these studies.

In this chapter, we shall review three major approaches to interpreting performance on the nine-dot problem: Gestalt theory, a retrieval/hypothesis model, and an information processing approach. We shall then discuss other controversial issues involving the nine-dot problem.

\section{Gestalt Theory}

Gestalt psychologists have been studying perception, fixation and insight in problem solving since nearly the beginning of this century. For Gestalts, nine-dot and similar kinds of problems are difficult because problem solvers place restrictions on their solutions to the problem. Removing these restrictions results in insight concerning how to solve the problem.

\section{Fixation}

Martin Scheerer (1963) proposed that individuals enter tasks with many restrictions or "fixations" on how to solve the problem. These fixations cause subjects to cling to false assumptions about the task. For example, subjects often display functional fixedness. Functional fixedness refers to the difficulty a problem solver has in using an object in a manner different from that to which he is accustomed. The effect was neatly illustrated by Duncker (1945) through his candle holding experiment. When the boxes were empty and lying loose, subjects were more likely to use the boxes as shelves for the candles to 
solve the problem of mounting the candles vertically on a wooden screen. When the boxes were not empty, subjects tend to respond to the boxes as containers (fixed function) but not as shelves for the candles. Other fixations include unwillingness to accept a detour that delays the achievement of one's goal and habitual modes of responding to situations. If one is presented with several objects in succession and the perceptual attributes of the later objects reflect the influence of earlier objects, a mental set is formed.

The response set effect was well illustrated by Luchins' (1942) water jar problems. His experimental results indicate that solving a number of problems by one method tends to trap the person into using the same method on later problems, even if the method becomes inappropriate. Because the well practiced bit of knowledge may be applied uncritically, practice can be carried to the point where it becomes an inhibiting factor in creative problem solving. When a person performs a habitual, well-drilled act, he is no longer coping with the problem (Krech \& Crutchfield, 1968).

Functional fixedness, unwillingness to accept detours, and response set might contribute to difficulties in solving the nine-dot problem in subtle ways but the literature has not addressed these influence on performance. Among various forms of fixation, inappropriate assumptions are considered most crucial in limiting performance on the nine-dot problem. 
According to Gestalt psychologists, inappropriate assumptions are obstacles to success. In the nine-dot problem, subjects assume that they must stay within the group of dots. This assumption is implicit, most people do not even know they have made it. Maier and Casselman (1970) tested 311 male and 233 female college students with six insight problems, including the nine-dot problem. Subjects were allowed 45 minutes to work on the six problems, and were encouraged to limit work to 5-8 minutes on difficult problems. According to the authors, the time allotted seemed to be adequate. There was no reluctance to turn in the paper at the end of the period and many seemed to have given up after half an hour. Subjects were presented with either the standard version of the nine-dot problem or with an altered version in which the dots were enclosed within a larger frame. Maier and Casselman reported that adding the frame resulted in more solutions to the problem. Going outside the area of the dots did not guarantee a solution, but facilitated solution. They observed a sex difference in the percentage of boundary breakers ( $51.9 \%$ males, $22.9 \%$ females) and problem solvers (42.0\% males, $17.7 \%$ females).

Maier and Casselman made two important inferences about their results: First, inappropriate assumptions hinder problem solving. Second, different individuals may perceive the problem situation differently. They suggested that chance factors play an important part in problem solving and that the tendency to avoid persistence in perception and to continue to seek alternatives 
seems to be a matter of ability. They also suggested that the factors that make a problem insolvable are not the same for all people. When exposed to a given situation, different persons would not be working on the same problem. Maier and Casselman's study (1970) was the first to address individual and sex differences in the nine-dot problem. But one problem for this study was whether subjects were ready to give up in their efforts to solve the nine-dot problem. Moreover, subjects worked on several problems in this study and they might have been generally tired.

The Gestalt view that the assumed boundary is disruptive to the solution of the nine-dot problem is shared by some non-Gestalt psychologists. For instance, Newell and Simon (1972) stated:

Most subjects adopt a representation lof the nine-dot problem) that assumes the straight lines must all terminate on the dots and cannot continue outside the boundaries of the square. With this restriction--imposed by the representation and not by the problem statement--the problem is insolvable. If the subject at any moment considers the possibility of generating lines that extend outside the square, he finds the solution very quickly. (p.91)

Glass, Holyoak and Santa (1979) wrote in Cognition: When people are given the (nine-dot) problem, they tend to assume that the four lines cannot go outside the imaginary boundary.... If problem solvers can modify their internal 
representation of the problem by eliminating this constraint, they may experience a feeling of insight.... Once these crucial steps have been made, the problem is, in a sense already solved. (p.404)

\section{Directions and Insight}

Maier (1930) used the nine-dot problem to illustrate how changing "direction" aids the solution of a problem. He pointed out:

This problem is difficult because all attempts are made within the area of the dots. To leave this area seldom suggests itself as a possibility. If it is suggested that one need not confine himself to the area within the square, a whole new field of possibilities presents itself. (p.142)

Maier (1940) discussed the difference between what he called habitual and new directions in The Behavior Mechanisms Concerned with Problem Solving:

Habitual directions are states in which the individual reproduces old solutions.... New directions are less specific in that they do not result in reproductive solutions but give rise to a new combination and hence new product.... (p.51)

\section{Retrieval/Hypothesis Model}

R. W. Weisberg and J. W. Alba (1981a) questioned the commonly accepted view that the nine-dot and triangle problems are difficult because subjects are fixated on unwarranted assumptions about how the problems are to be solved. They conducted a series 
of experiments to examine the role of fixation (a Gestalt concept) in the solution of these insight problems. They used undergraduate students as subjects to see whether quick and direct solutions of the problem would happen after eliminating the allegedly fixating assumptions. The subjects working on the nine-dot problem were told that the problem could be solved only by extending their solution lines outside the boundaries of the square formed by the dots. The authors reported that removing the alleged fixation did not result in sudden and direct solution of the problem. They argued that fixation was not a very important factor in making the problem difficult and that significant facilitation could be brought about only by giving subjects relatively detailed information about the solution. They concluded that the term fixation and insight are not useful in describing the processes involved in the solution of the nine-dot problem and its variant such as the triangle problem.

As an alternative, Weisberg and Alba proposed a retrieval model. This model incorporates ideas from Weisberg and Suls (1973) and Levine (1975). In this framework, the presentation of a problem serves as a cue to retrieve relevant information from long-term memory. Information that is retrieved then serves as the bases for solution attempts. Hence, problem solving begins with relevant past experience. However, subjects do not simply apply old solutions directly to new problems. They monitor these solutions, and if they are not working, they try to modify the inadequacy so that the solution becomes appropriate. For the 
nine-dot problem, people tend to go from dot to dot based on their recall of past experiences with puzzles and games. When this method does not work, they must try further modifications. Unfortunately, most people do not have additional knowledge of how to modify a dot-to-dot solution to solve the problem.

As Weisberg and Alba (1981a) stated, the retrieval model is similar to Levine's (1975) hypothesis model which views solutions to problems as consisting of groups or "domains" of solutions. Different solution domains will be sampled based on the knowledge and assumptions that the subjects bring to the problem. Difficulty in insight problems occurs for four reasons: a) The presentation of the problem in conjunction with the subjects' past experience suggests one way of attacking the problem (i.e., suggests one domain of solutions). b) This domain is relatively large so that subjects may never exhaust it. c) Even if subjects do exhaust the initial domain, the problem may still be difficult because they may not be aware that another domain of solutions exists due to lack of relevant past experience. d) Even if subjects do believe that an alternative domain exists, the domain may also be so large that subjects who have to work through the possibilities may still not solve the problem. (p.172)

As to why the control subjects in the experiments could not solve the problem, Weisberg and Alba theorized: It seems that the domain which contains the correct solution effectively does not exist, perhaps because individuals do not have relatively specific past experience that can be applied to the problem at 
hand. The reason subjects stay within the borders of the figure in the nine-dot problem may be that their past experiences direct them to go from dot to dot, which result in their staying within the figure.

Weisberg and Alba's work attempts to bring the study of insight problems into the mainstream of cognitive psycholology. They proposed a clearly-conceptualized alternative framework for understanding insight problem solving. They emphasized the importance of problem specific experience in insight problem solving and the results of their study indicate that appropriate past experience or training can facilitate insight problem solving.

\section{Information Processing Approach}

Lung and Dominowski (1985) felt that the retrieval/hypothesis testing framework proposed by Weisberg and Alba (1981a) and the Gestalt theory are inadequate and that an information processing approach is more appropriate. According to their viewpoint, success in solving insight problems requires appropriate representations of the task environment in subjects' problem spaces and the application of strategies which execute effective evaluation functions (Simon, 1978). A subject's usual strategy for trying to solve the nine-dot problem will include several steps: 1) Selection of a dot as a starting point, i.e., one of the four corner dots, one of the four dots in the middle of the edges, or the center dot, 2) evaluation of the available lines, i.e., whether a line is legal, optimal or new, 3) selection of a 
line based on the outcome of the evaluation, and 4) selection of a new starting point. Subjects repeat Step Two to Four until they successfully draw four lines or realize that they can not connect all the dots by the lines they have drawn.

An appropriate strategy for solving the nine-dot problem requires that the lines go outside of the boundaries of the square; a line must extend beyond the last dot in that line. The endpoint of this extension must form the intersect for the line to be drawn next. The difficulty of the nine-dot problem lies in subjects' representation of the problem as requiring lines starting and ending on dots. If so, the arrangement of the dots within a pattern should have a direct effect on the representation of the problem and, consequently, the strategy applied to solve the problem. Lung and Dominowski expected that if the subjects learned the proper representation and strategy, they should be able to apply these to the nine-dot problem and overcome the difficulty caused by the shape of the pattern.

Lung and Dominowski (1985) designed a study to investigate subjects' usual approaches to solving the nine-dot problem. They also examined whether instructing subjects to use a strategy of extending and intersecting lines outside the dots is effective, and, if subjects can acquire strategies appropriate for solving the problem by experience in solving other similar problems. They found that both instructions and practice facilitated nine-dot problem solving. Moreover, extending (even wrong) lines early and persistently was important for solving the problem. Among 
subjects who extended a correct line, $68 \%$ found the solution.

They interpreted this finding as a support of their proposal that the nine-dot problem belongs to a class of problems with similar strategic requirements. The quick solvers used $0-8$ extra trials, others used 12-36 extra trials to reach the solution. This result was contradictory to Weisberg and Alba's (1981a) proposal that facilitation requires knowledge of specific solution lines.

Lung and Dominowski reported an overall sex difference in the percentages of solvers of the nine dot problem $(40 \%$ for males and 21.9\% for females). They also found that subjects' answers to questions about their solution process provided no useful information, but examination of the lines subjects drew was informative. They concluded that, since the nine-dot problem has visual and motor components, it is possible that evaluations involving nonverbal encodings occur so rapidly that subjects are not fully aware of them. They reported some typical strategies in the way subjects drew the lines. For example, on the first trial, nearly all subjects selected one of the corner dots as the starting point $(90.6 \%)$, and about $95 \%$ of all first lines covered three dots, $83 \%$ of all second lines covered two dots. Thus, the first two lines subjects drew included the most dots. Additionally, they called attention to the process of evaluation of intermediate solution attempts, that may encourage efforts to trace the steps of the subjects in attacking the problem, and the course of the problem solving process. 
Lung and Dominowski's study is limited in several ways. The time limit and the way they used the timer (beep at 20 th second to warn subjects that 10 seconds were left) may have introduced an extraneous variable (interruption) to the experiment and could have had a disruptive effect on the problem solving process. Subjects were also asked to draw a solution attempt on a worksheet. If the solution was incorrect, the sheet was taken away and a new sheet was presented for another attempt. Using this procedure, the subjects were often distracted and deprived of the opportunity to review their products visually. Given all these factors, it is difficult to interpret the data. The rate of successful solvers might be under-estimated for all groups due to the ways this task was given.

\section{Controversial Issues}

\section{Are "Fixation" and "Insight" Useful Concepts?}

Weisberg and Alba (1981a) stated, in their empirical study of the nine-dot and triangle problems, that the terms "fixation" and "insight" are not useful in describing the process involved in the solution of these problems. They argued that fixation and insight are not really explanations but are merely descriptions. In addition, there may be circularity involved in the use of these terms. Moreover, Weisberg and Alba indicated that in both the nine-dot and the triangle problems, removing the alleged fixation did not result in all of the subjects easily solving the problem. Insightful solutions did not come quickly and directly. Their subjects also had difficulty recalling the insight 
solutions. Additionally, the term fixation has negative connotations: One is fixated when one keeps doing something that one ought to stop doing. Weisberg and Alba criticized Gestalt psychologists for playing down the positive role of past experience.

Dominowski (1981) disagreed with Weisberg and Alba's viewpoint. He tried to clarify and defend the position of Gestalt psychology. He argued: (1) General transfer effects in problem solving had not yet been adequately studied and problem solving is not necessarily restricted to direct transfer of past experience. (2) The term "fixation" does suggest something more than mere consideration of an idea; it connotes obsession or an excessively strong adherence to an idea. If some means could be found to distinguish varying degrees of adherence to ideas, then fixation could serve some useful theoretical purpose.

Dominowski argues that "Insight" is used in several different ways. On some occasions, insight does seem to be used to refer to an exotic process that somehow produces solutions to problems. This usage should be avoided. Weisberg and Alba equate "insightful" with fast, and this usage should also be avoided. But sometimes insight refers to an idea, that, if apprehended, might be necessary, sufficient, or advantageous for solving the problem. "Insight" sometimes refers to problem solvers' awareness of how a solution was reached or why a particular approach yields a satisfactory solution. The latter meanings for insight refer to some forms of understanding. In these senses, insight can serve a 
central role in the development of improved theories of problem solving.

Weisberg and Alba (1981b) countered that the use of fixation to distinguish varying degrees of adherence to ideas was not relevant to subjects who cannot solve the nine-dot problem and who never thought of going outside the dots. These people were not excessively adhering to any idea, they simply did not know what to do. Weisberg and Alba suggested that the use of insight to refer to the problem solver's awareness of a solution could be replaced by the term understanding. According to Weisberg and Alba, the use of insight as an idea, is unnecessary except in Gestalt theory. In the nine-dot problem, if one does not assume fixation on the assumption that the lines must stay within the dots, then the awareness of going outside the dots is not a particularly crucial bit of information.

Dominowski responded to Weisberg and Alba's refutation with a study reporting (Lung and Dominowski, 1985) that extending Iines early and persistently is important for solving the problem and that their data contradicted Weisberg and Alba's (1981a) proposal that facilitation requires knowledge of specific solution lines.

In summary, fixation and insight are important concepts in Gestalt psychology. Psychologists disagree, however, on whether these concepts are useful today and how useful they are. To answer this question, further studies are needed. 
What IS the Nature of the Difficulty of the Nine-Dot Problem?

Nearly all the studies we have reviewed have addressed the nature if the difficulty of the nine-dot problem. This difficulty has been explained in several ways. According to Kendler (1963), perception plays a vital role in problem solving but its role is not clearly distinguishable from that of learning. He pointed out:

The very way a problem is presented elicits perceptual responses that retard problem solution.... In the nine-dot problem the dots are perceptually grouped together to form a square. As a result, the subjects' tendency to extend his line beyond the limits of the square is very weak.... Perceiving the dots as a square makes him respond to them as a square. (p.370)

He summarized as follows:

In short, the manner in which we perceive a problem determines the relative strengths of different response tendencies. When our perceptions direct us toward making the correct response, problem solution is hastened. When our perceptions increase our tendency to make inappropriate responses, the problem solution is retarded. (p.371) ... the difficulty of a problem depends upon how it is perceived. (p.372)

Kendler's words reflect the "perception-difficulty" view. Maier and Casselman (1970) found that failures in most of the problems used in their study were caused because the 
presentations of the problem were not appropriate to the subjects' perception. Lung and Dominowski (1985) reported that their main study results stress the importance of an appropriate strategy but their other findings indicate that perceptual factors should not be ignored.

Weisberg and Alba (1981a) concluded that the difficulty of the nine-dot problem may be due to other than perceptual effects. The shape of the dots did not "hold" the subjects within it. Rather, the reason subjects stayed within the square on the nine-dot problem is simply they went dot to dot (p.182).

Twelve years before Weisberg and Alba's study, C. A. Burnham and K. G. Davis (1969) conducted a study to identify difficulties contributing to the nine-dot problem and reported these difficulties were beyond perceptual organization. They administered twelve versions of the nine-dot problem to 194 college students with a time limit of ten minutes. They found that instructions stating that subjects could extend the lines beyond the boundary of the square formed by the nine dots were marginally effective in improving performance. Meanwhile, they reported that presenting two extra dots outside the square (see APPENDIX C) dramatically increased the number of solutions. The presentation of extra dots resulted in significantly more solvers than the instructions stating that the lines could extend beyond the square formed by the dots. This finding partially supports the perceptual organization interpretation of difficulty of the nine-dot problem by Kendler (1963). 
Burnham and Davis' interpretation of the findings was as follows: The verbal instruction may or may not alter the perceptual organization. Such instructions do cause subjects to extend their lines beyond the boundary of the square. But doing so does not usually result in solving the problem. The presentation of eleven dots probably provided a direction and a rationale for drawing the neccessary four lines for this solution. They argued: a) Perceptual organization (seeing the dots as confining a square) is a minor factor in making the nine-dot problem a difficult one. b) The source of the difficulty is that the sequence of lines involved in the solution is not obvious.

Can we conclude that perceptual organization is a minor factor merely because instructions that lines may go outside the dots were marginally effective in improving performance, and the presentation of extra dots resulted in significantly more solvers than the instruction procedure?

Are there other possible difficulties beside the none obvious sequence of Iines?

These two questions come from the following considerations: a) The triangle shape of the eleven dots is exactly the shape of the solution. It may give a graghic or perceptual hint to the solution. In contrast, the square of the nine dots gives a misleading hint. Hence, the effect of the stimulus pattern on perceptual organization may play an important role in facilitating eleven-dot problem solving as well as in disrupting 
nine-dot problem solving. b) The usual dot-to-dot connecting strategy or the cover-as-many-dots-as-a-line-can strategy is effective for the eleven-dot problem but not for the nine-dot problem. Therefore, to solve the nine-dot problem, one needs a new strategy. c) If the subjects assume that the solution should not go beyond the boundary formed by the dots, or, that the intersection of the lines should be on a dot, they can still solve the eleven-dot problem but they will not solve the nine-dot problem.

Now, let us discuss the methodology of Burnham and Davis' study. In the study, they introduced twelve versions of the nine-dot problem. Series 1--standard form: 1A, no additional instructions; 1B, outside permitted; 1C, crossing and touching permitted; 1D, outside and crossing and touching permitted. Series 2--start at lower left plus the same four sets of instructions used in Series 1 . Series 3--11 dots. Two additional dots were presented in the Series 3 versions. Two sets of instructions were used: $3 \mathrm{~A}$, no additional instructions; $3 \mathrm{~B}$, crossing and touching permitted. Series 4--11 dots and start at lower left. The same two sets of instructions used in Series 3 were used. These twelve versions provided valuable information on the relationships between different types of instructions. They could help us pinpoint the effects of various assumptions on the performance of the problem solving.

Unfortunately, this study shares some of the weaknesses with the ones we reviewed earlier, i.e., using a convenient sample 
(college students) and giving Iimited time (10 minutes). Besides, the subjects were told to erase their presolution attempts. This procedure takes time from the allotted ten minutes and eliminates the visual availability of previous solution attempts.

\section{Summary of Literature Review}

In this chapter, we have reviewed three major approaches to explaining the difficulty individuals have in solving the nine-dot problem. Gestalt psychologists think the assumed boundary plays a crucial role in insight problem solving. Some cognitive psychologists do not believe that this fact plays a prominant role in explaining performance. Among the few empirical studies done on the nine-dot problem, some report that their data support the proposal that breaking the assumed boundary is crucial to nine-dot problem solving (e.g., Lung \& Dominowski, 1985), some report that their data contradict this explanation (e.g., Weisberg \& Alba, 1981). There are reports on individual and sex differences in the problem solving. One study reported that subjects' drawings of the solution were more informative than verbal protocols in the study of nine-dot problem solving.

The work to be done includes finding answers to important questions such as "How universal is the effect of the assumed boundary in nine-dot problem solving across age, sex and culture?" To answer this question, we need a well designed crosscultural and developmental study of performance on the nine-dot problem. 
CHAPTER 3

METHODOLOGY

\section{Introduction}

The purpose of this study was to provide a broader range of evidence for the universality of the effect of the assumed boundary in solving the nine-dot problem. To achieve this goal, two cultural (mainstreamed Chinese and mainstreamed American) and four age groups $(6-7,10-11,15-16,17-18)$ were tested. These four age groups correspond to late preoperational, late concrete operational, formal operational and late formal operational levels in Piaget's theoretical framework. Cultural, developmental stage and sex differences can be expected to affect performance on the nine-dot problem as the following rationale suggests:

1. Cognitive ability generally develops throughout the age range included in this study. Higher cognitive ability is often a hallmark of greater capability in solving complex problems. Subjects at higher ages can be expected to do better in the nine-dot problem than subjects at lower ages.

2. Maier and Casselman (1970) studied individual and sex differences in nine-dot problem solving. They found that males $(51.9 \%)$ were more likely to go outside the area of the dots than females $(22.9 \%)$ and this accounted for males' greater success of the problem $(42.0 \%)$ than females' $(17.7 \%)$. Lung and Dominowski (1985) also reported an overall sex difference in the percentages 
of solvers of the nine-dot problem $(40 \%$ for males and $21.9 \%$ for females). However, no explanation was offered for the sex differences found in these studies.

Sex differences in the performance on the nine-dot problem may stem from gender differences in cognitive style. Field independents have the ability to separate parts from the whole while field dependents perceive the field holistically as a unit. Females tend to be slightly more field-dependent than males (Berthelot, 1982; Mwamwenda, Dionne \& Mwanwenda, 1985; Chadha,1985). Lin (1982) found that high-school subjects characterized as field independent performed better on formal-operational tasks than those who were field dependent. Males not only had higher scores than females on measures of field independence, but also on measures of propositional logic and combinatorial reasoning. Loader, Edwards and Henschen (1982) reported that more field-independent male basketball players were able to change perceptual set. There may be consistent differences between males and females in ability to change perceptual set. Swinnen (1984) explained the higher learning rate of field independent boys in terms of information processing systems, suggesting that the use of analytic and structural mediators in operating on complex stimulus configurations leads to more differentiated movement images.

Interactions between culture and sex have been found with blacks, whites, and Mexican Americans (Chang, 1984). Maltese-Australian females have been found to be significantly 
more field dependent than their male counterparts (Gauci, 1983). Pandey and Pandey (1985) found males were more field independent than females and, urban males and females were more field independent than their rural counterparts. Fowler and Fowler (1984) found Nigerian school children were more field dependent than the undergraduates. Chatterjea and Paul (1982) found significantly more field independence and geometrical figure recognition capacity among urban subjects than among rural subjects. There were no significant sex differences between the the urban and rural goups on these two variables.

3. Chinese and American cultures are strikingly different in language, history, social system, social norm, basic values, child rearing and educational systems. China is an agricultural country with 1.1 billion people and a long history. Her people have been in this land for thousands of years. Stability, unitary and centralized power are some of the characteristics of this nation. The United States is a young industrialized country with 200 million people. Her population consists of immigrants from all over the world. This young nation is marked by mobility, diversity and decentralization of power. As a result of this cultural diversity, the social norms in the United States are much less clear-cut and restrictive than in China. American society is less demanding of conformity from children or adults.

Individualism is valued in the United States. This value is reflected in child rearing practices. In American middle-class homes, the emphasis on independence or separateness begins at 
birth. The infant is often kept in a separate room. Babies have their own space and belongings which increase as they grow older. Children are seen as individuals and are expected to have their own choice or preference for things. Children in China are brought up to merge with others. From birth through five years of age, and sometimes as even older children, they share a bedroom with their caregivers, usually parents or grandparents. They do not have their own space. They are not seen as individuals but a member of the family. They are expected to be obedient to adults.

American children experience diversity, autonomy and individualism in their schools. Children in different schools within a town may have very different curriculum. Within a school, a child may be in an open setting in one classroom while the child next door may be enrolled in a traditional teacher-centered classroom. Within a class and within the same hour, students are often working on different tasks and doing different things. Individualized education allows students to progress at their own pace. Chinese children study in schools that are under highly centralized leadership which decides requirements for teachers, curricula and textbooks for the whole nation. To be in the same class means to study with the same teachers, to do the same tasks at the same time, and to have the same academic requirement and homework. Collectivism, hard work and self-discipline are emphasized beginning in the the first grade. 
Mobility, autonomy, tolerance of diversity and respect for the individual may create an environment that nurtures exploring behavior which is necessary for creative problem solving.

In China, walls or fences typically bound any estate including homes, schools, universities and factories. One has to enter or exit through a gate. In the United States such walls are scarcely seen.

On the other hand, the chinese children tend to receive more training in mathematics which may facilitate performance on the nine-dot problem. Students, including first graders, study in school six days a week, 10 months a year. They have fewer holidays and much more homework than their American counterparts. From elementary to senior high school they have mathematic exercises almost every day. By the end of the fourth grade, Chinese students have gained knowledge about basic shapes and how to compute their area. After eight years of schooling, they have learned basic plane geometry. A good understanding of the concept of straight line may help one to comprehend the direction of connecting the nine dots with four straight lines. By the twelfth year of schooling, students have learned solid geometry and trigonometry. By the end of the twelfth year schooling they have reviewed all the mathematics they have learned. This emphasis on training in geometry may provide students with knowledge and strategies which facilitate the solving of the nine-dot problem. But the emphasis on mathematic skill training 
may also be disadvantageous. Well practiced knowledge may be applied uncritically to solve the nine-dot problem.

According to teachers and participants in this study, similar basic geometric concepts are learned in the two cultural groups at each age level, but the Chinese children have more exercises involving math concepts and skills.

In summary, participants from both cultures have advantages and disadvantages. Thus, it is difficult to predict which group should do better on the nine-dot problem although cultural differences can be expected to contribute to performance. Based on the review, it was predicted: (1) there would be cultural difference in number of boundary-breakers and problem solvers and time spent on the problem, (2) the older age groups would have more boundary-breakers and problem-solvers, and take less time to solve the problem than the younger age groups, and (3) males would do better in breaking the boundary and solving the problem than females.

\section{Sample}

Twenty mainstreamed Chinese from Beijing and 20 mainstreamed Americans from Massachusetts in each of four age groups (6-7, $10-11,15-16,17-18$ ) were included in this study. The total number of participants was 160 , half in each group were males and half were females. The procedures for sampling were the following:

The Chinese sample was drawn from both key and regular schools in Beijing. Key schools have more highly qualified 
teachers, better equipment and students with better academic records and higher scores on entrance exams than regular schools. one third of the students came from key schools and two thirds from regular schools. In each school, there were several classes in each grade. Each class in an elementary school consisted of 35-40 pupils, and each class in a high school consisted of 45-55 students. A key elementary school and a key high school, a regular elementary school and a regular high school participated. Administrators permitted the experimenter to select participants from a class by using random numbers. She called odd or even numbers on the students roll.

In the first stage of data collection, the experimenter was surprised at the high percentage of 6-7 year olds from a Chinese elementary school who were problem solvers (67\%) within the relatively short time of 30 minutes on average. Through inquiry she learned that these pupils had participated in a course of thinking skills. To eliminate this potential biasing experience, she excluded this group from the sample and collected data from a more typical sample of children.

The American sample was drawn from the mainstreamed American population in both a university community and a working-class community. One elementary school and one high school were chosen from a university town. One elementary school and one high school were also chosen from a nearby working-class community. The pool of subjects available from the four American schools was smaller than that from the Chinese schools. There were about 40-50 
children in each age group of each high school and $20-40$ children in each age group of each elementary school. Half of the 10-11 year old participants were taught by one teacher who emphasized strategies of problem solving in her math class. Administrators in each school were also asked to choose participants randomly. One high school principal chose the 20 participants at age $15-16$ and 17-18 through odd or even numbers. The other high school and the two elementary schools included classes of the corresponding age groups in the nine-dot task. The experimenter randomly chose the worksheets (which were numbered) by first shuffling them and then taking all the odd numbers.

The Chinese participants completed the task in classrooms after school. The American participants completed the task in classrooms or a teachers' office during school time. Both groups showed considerable interest in the problem. For example, Some students continued to work on the problem at home. Some discussed the solution with peers and teachers after they handed in their worksheets.

Participants were students within normal classrooms. No learning or mentally disabled students were included in the sample. All children who reported having done the task before were excluded from the data collection.

\section{Task}

The task was the nine-dot problem. Participants were given instructions to connect the nine dots by drawing four straight lines without retracing and without removing their pencil from 
the paper. The dots were graphed on $8 \times 11$ in. sheets. Each participant was presented 6 worksheets, with six numbered graphs of the nine-dot problem on each worksheet (see APPENDIX A).

\section{Procedures}

The task was completed individually. Before giving the task, the experimenter read the directions on the worksheet to the participants (see directions in APPENDIX A). The experimenter emphasized that she was interested in every trial.

The time the participants started, the time the participants extended the square boundary, and the time they took to solve the problem, or gave up (see the time recording sheet on APPENDIX $B$ ) were recorded. Subjects did not perform under any experimenter -induced time limits. However, the ideal of letting each participant solve the problem at his own pace was constrained by a number of factors not under the control of the experimenter. For example, after 130 minutes the $10-11$ year old Chinese pupils still working on the problem had to stop because teachers and parents could not wait for them more than two hours after school. When observing others going to their next class or preparing to leave school, the American participants in the high school grades may have also felt under pressure to end their efforts to solve the problem.

\section{Research Design and Data Analysis}

The independent variables were culture, age and sex. The dependent variables were (a) number of solution attempts before extending the boundary formed by the nine dots; (b) number of 
solution attempts before solving the problem; (c) total number of solution attempts before terminating problem solving effort; (d) time spent before extending the boundary formed by the nine dots; (e) time spent before solving the problem; (f) time spent before terminating problem solving effort. In addition, the number of boundary breakers and the number of solvers in each group were computed and compared. The relationship between having the shape of the solution within the boundary and solving the nine-dot problem was also analyzed.

A summary of the 2 (cultural groups) $\times 4$ (age groups) $\times 2$ (sex groups) design is provided in Table 3.1 .

A three factor multivariate analysis of variance was used to analyze the six dependent variables. A Chi-square test was used to compare the number of solvers in each group. The accepted $\mathrm{p}$ level was .05 to reject the null hypotheses. 
Table 3.1

A Summary of the Design for the Analysis of Variance

\section{Cultural Groups}

Chinese American Total

$\operatorname{Sex}$

Ages $\quad M \quad F \quad M \quad F$

$6--7 \quad 10 * 10 \quad 10 \quad 10 \quad 40$

$\begin{array}{llllll}10--11 & 10 & 10 & 10 & 10 & 40\end{array}$

$\begin{array}{llllll}15--16 & 10 & 10 & 10 & 10 & 40\end{array}$

$\begin{array}{llllll}17--18 & 10 & 10 & 10 & 10 & 40\end{array}$

$\begin{array}{llllll}\text { Total } & 40 & 40 & 40 & 40 & 160\end{array}$

* = Number of subjects in each cell. 
CHAPTER 4

RESULTS

This study was designed to examine cultural, developmental and sex difference in performance on the nine-dot problem solving. The results of the study are summarized as follows:

\section{Observations}

The contrast of approaches to the task of the two cultural groups was very interesting: Most Chinese students focused on what was required of them and spent more time on understanding the rules than their American counterparts who were ready to start quickly and rapidly completed trials. On the average Americans made 1.52 trials per minute while their Chinese counterpart only made 0.85 trials per minute. It was noticed by teachers that the Chinese students referred to the directions now and then in the problem-solving process.

It should be noted that substantial individual differences were also observed. These differences are evident in the amount of time spent on the problem and the number of trials completed. The shortest time taken to solve the problem was less than one minute but the longest time was 145 minutes. The shortest time unsuccessful subjects worked on the problem was seven minutes and the longest 130 minutes.

Cross-cultural, cross-age and cross-sex similarities in attacking the problem were also observed. Almost all of the participants started from one side and tried to cover as many 
dots as they could with one line. When subjects failed to connect the nine dots within the boundary after a series of trials, some gave up, but others began to extend their Iines outside the boundary. When this occurred, $65 \%$ of the subjects solved the problem.

\section{Hypothesis 1 Culture Effect}

Chinese students were more likely than American students to solve the problem. Fourty-three out of 80 Chinese students solved the problem but only 17 out of 80 American students solved the problem, a highly significant difference $\left(\mathrm{X}^{2}=18.03, \mathrm{~d} f=1\right.$, p<.001). Fifty-five Chinese students and 38 American students went beyond the boundaries. This difference was also reliably different $\left(X^{2}=7.42, d f=1, p<.01\right)$. The results revealed that $78 \%$ of the Chinese boundary-breakers solved the problem while $45 \%$ of the American boundary-breakers solved the problem.

The Chinese children spent a longer time $(p<.05)$ on the problem $(32 \mathrm{~min}$. to break the boundary, $41 \mathrm{~min}$. to solve the problem, and $75 \mathrm{~min}$. before giving up) than the American group (21 min. to break the boundary, $26 \mathrm{~min}$. to solve the problem, and $30 \mathrm{~min}$. before giving up). The average time between breaking the boundary and solving the problem was nine minutes for the Chinese group, five minutes for the American group.

In trials made to break the boundary, the two groups were very close ( 33 for Chinese, 30 for American). In trials to solve the problem, the 6-7-year-old groups were excluded because no one in the 6-7-year-old American group solved the problem. The 
average number of trials to solve the problem were 39 for the Chinese and 29 for the American group. Although the average number of trials made by unsuccessful problem-solvers looked close (83 for Chinese, 68 for American) the frequency distribution of the two groups was different (see Figure 4.1). The typical American participants in all four age groups gave up after 40-45 trials. In contrast, their 6-16-year-old chinese counterparts gave up after $40-120$ trials. The five unsuccessful 17-18-year-old Chinese students terminated their efforts after 20-42 trials.

A multivariate test demonstrated significant cultural differences $(p<.05)$ for time and trials before breaking the boundary, to solve the problem, and before giving up (see Table 4.1, Table 4.2 and Table 4.3). A univariate test (see Table 4.4) revealed that the major source of difference came from the time spent on the task, but not the number of trials. 

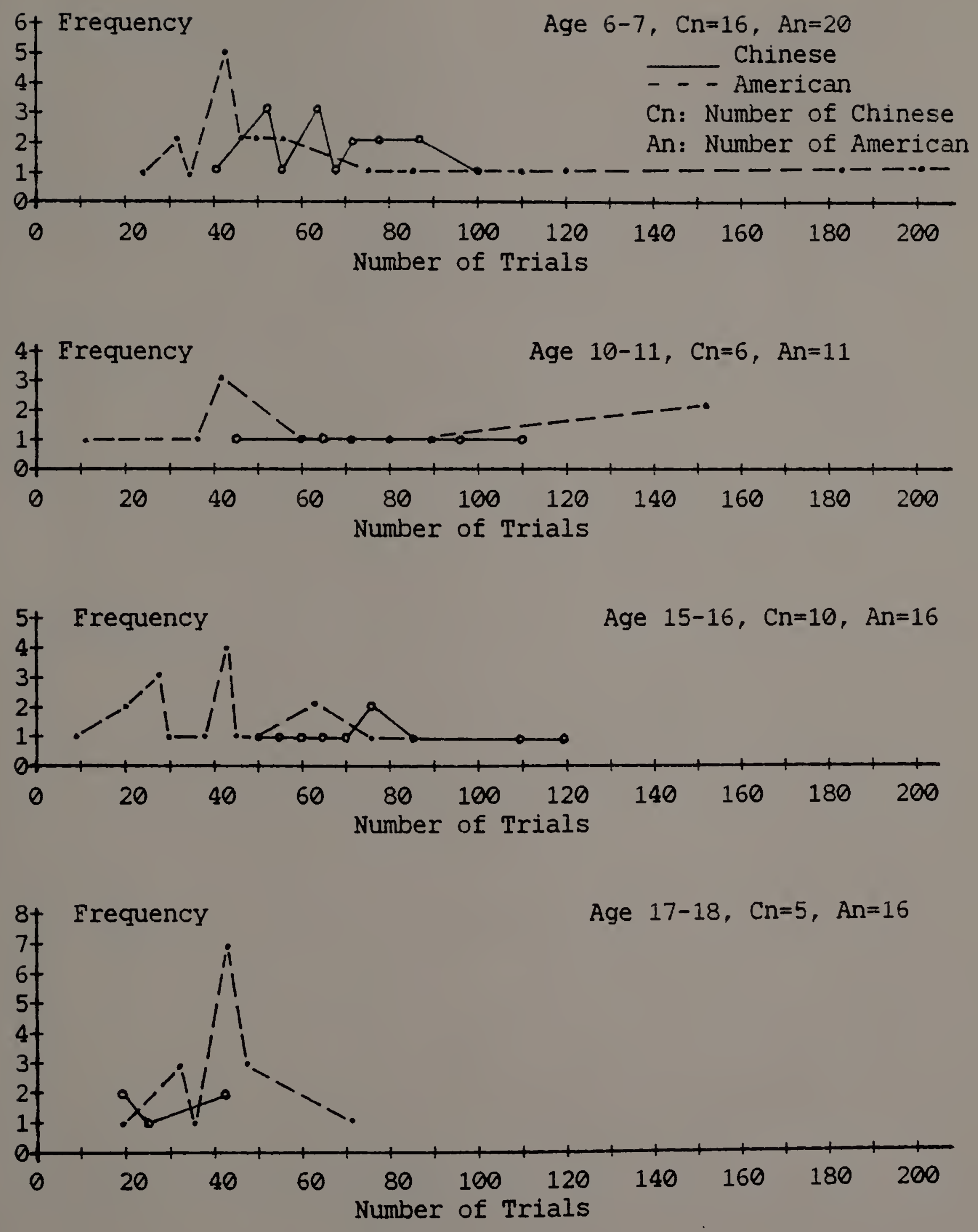

Figure 4.1

Frequency Distribution of Trials on the Problem by Unsuccessful Solvers 
Table 4.1

Multivariate Tests of Significance

-- Time and Trials to Solve the Problem

\begin{tabular}{llccccc}
\hline Effect & Test Name & Value & Exact F. Hypoth.DF & Error DF & Sig.of F. \\
Cult. & Wilks & .81094 & 5.01244 & 2.00 & 43.00 & $.01105^{*}$ \\
Age & Wilks & .72920 & 2.45181 & 6.00 & 86.00 & $.03090^{*}$ \\
sex & Wilks & .94865 & 1.16373 & 2.00 & 43.00 & .32196 \\
C*S & Wilks & .86790 & 3.27258 & 2.00 & 43.00 & $.04754^{*}$ \\
C*A & Wilks & .89714 & 1.19910 & 4.00 & 86.00 & .31712
\end{tabular}

Note: Cult $=$ Culture effect; $C^{\star} S=$ Interaction between culture and sex; $C^{\star} A=$ Interaction bwtween culture and age.

* means $p<.05, * *$ means $p<.01$ after adjustment.

Age* Sex and Cult*Age*Sex were not included in the test and were not significant, thus are not reported in the MANOVA tables.

Table 4.2

Multivariate Tests of Significance

-- Time and Trials to Break the Boundary

\begin{tabular}{lllcccc}
\hline Effect & Test Name & Value & Exact F. Hypoth.DF & Error DF & Sig.of F. \\
Cult. & Wilks & .87693 & 5.40340 & 2.00 & 77.00 & $.00637^{\star *}$ \\
Age & Wilks & .76462 & 3.68595 & 6.00 & 154.00 & $.01370^{\star}$ \\
Sex & Wilks & .96894 & 1.23405 & 2.00 & 77.00 & .29680 \\
C*S & Wilks & .93096 & 2.85536 & 2.00 & 77.00 & .06364 \\
C*A & Wilks & .86542 & 1.29365 & 6.00 & 154.00 & .08030 \\
\hline
\end{tabular}

Note: Cult $=$ Culture effect; $C^{\star} S=$ Interaction between culture and sex; $C^{\star} A=$ Interaction bwtween culture and age.

* means $p<.05$, ** means $p<.01$ after adjustment.

Age ${ }^{\star}$ Sex and Cult*Age ${ }^{*}$ Sex were not included in the test and were not significant, thus are not reported in the MANOVA tables. 
Table 4.3

Multivariate Tests of Significance

-- Time and Trials by Unsuccessful Solvers on the Problem

\begin{tabular}{|c|c|c|c|c|c|c|}
\hline Effect & Test Name & Value & Exact $F$ & Hypoth.DF & Error DE & Sig.of E. \\
\hline Cult. & Wilks & .28445 & 104.394 & 2.00 & 83.00 & $\theta^{* *}$ \\
\hline Age & Wilks & .42471 & 14.7866 & 6.00 & 166.00 & $\theta * *$ \\
\hline Sex & Wilks & .99025 & .40870 & 2.00 & 83.00 & .66584 \\
\hline$C^{\star} S$ & Wilks & .96070 & 1.69787 & 2.00 & 83.00 & .18937 \\
\hline$C^{\star} A$ & Wilks & .50134 & 11.4075 & 6.00 & 166.00 & $0 \star \star$ \\
\hline
\end{tabular}

Note: Cult $=$ Culture effect; $C^{\star} S=$ Interaction between culture and sex; $C^{\star} A=$ Interaction bwtween culture and age.

* means $p<.05, * \star$ means $p<.01$ after adjustment.

Age ${ }^{\star}$ Sex and Cult*Age* Sex were not included in the test and were not significant, thus are not reported in the MANOVA tables.

Table 4.4

Summary of Univariate $F$ Tests on Cultural Effect

\begin{tabular}{lcccc}
\hline Variable & Hypoth.DF & Error DF & F & Sig. of F \\
B-time & 1 & 78 & 5.8562 & $.01785^{\star}$ \\
B-trial & 1 & 78 & 0.0881 & .76736 \\
S-time & 1 & 44 & 8.7436 & $.00498^{\star *}$ \\
S-trial & 1 & 44 & 2.8020 & .10124 \\
& & & & \\
G-time & 1 & 84 & 157.2651 & $0^{\star \star}$ \\
G-trial & 1 & 84 & 3.6207 & .06049 \\
\hline
\end{tabular}

Note: B-time=time spent until breaking the boundary.

B-trial=trials made until breaking the boundary.

s-time=time spent to solve the problem.

s-trial=trials made to solve the problem.

G-time=time spent before giving up.

G-trial=trials made before giving up. 


\section{Hypothesis 2 Age Effect}

Within each cultural group, the differences in the number of problem-solvers among the four age groups were significant (see Table 4.5, p.45. $X^{2}=15.03, d f=3, p<.01$ for the Chinese; $X^{2}=12.18$, $d f=3, p<.01$ for the American). For the Americans age differences were also significant for the number of boundary-breakers $\left(\mathrm{X}^{2}=\right.$ 15.44, $d f=3, p<.01)$. However, the difference among the Chinese groups in boundary-breaking was not statistically significant $\left(\mathrm{X}^{2}\right.$ $=4.83, \mathrm{df}=3, \mathrm{p}>.05)$.

From Table 4.6 (p.46) and Table $4.7(\mathrm{p} .47)$ we can see a trend across the two cultures for time and trials needed to solve the problem decreased with age. This is also true in the time and trials needed to break the boundary for the American group. For the Chinese, the three younger age groups required similar times before breaking the boundary, however, the 17-18 year old group required only about one half the time of their younger counterparts.

A multivariate test revealed that the effect of age was significant ( $p<.05$; see Table $4.1,4.2$ and 4.3 ) for the amount of time spent and number of trials required to break the boundary, to solve the problem, or before giving up. A univariate test indicated a highly significant age difference $(p<.01)$ in both time and trials to break the boundary, before giving up, and age differences were also highly significant for time to solve the 
probiem (see Table 8, p.48, after adjusting, p>.05 for the significance of difference in trials to solve the problem).

Table 4.5

Number of Subjects Producing Lines Extending beyond the Boundary and Solving the Problem

\begin{tabular}{ccccc}
\hline & \multicolumn{2}{c}{ American } & \multicolumn{2}{c}{ Chinese } \\
& Solver & B-Breaker & Solver & B-Breaker \\
$6-7$ & 0 & 7 & 4 & 12 \\
$10-11$ & 9 & 17 & 14 & 16 \\
$15-16$ & 4 & 8 & 10 & 11 \\
$17-18$ & 4 & 6 & 15 & 16 \\
& & & & \\
\hline \multirow{2}{*}{$* 17$} & 17 & 38 & 43 & 55 \\
\hline
\end{tabular}

Note: Solver $=$ subjects who solved the problem.

B-Breaker = subjects who broke the boundary. 
Table 4.6

The Average Time Spent by Each Age Group

to Break the Boundary, to Solve the Problem, and before Giving Up

\begin{tabular}{rrrrrrrrrrrr}
\hline & $\mathrm{n}$ & B-time & SD & n & S-time & SD & n & G-time & SD \\
$6-7$ & 12 & 35.35 & 10.40 & 4 & 75.50 & 26.17 & 16 & 58.69 & 8.45 \\
C $10-11$ & 16 & 39.26 & 29.71 & 14 & 57.58 & 29.24 & 6 & 130.0 & 0 \\
$15-16$ & 11 & 34.64 & 25.32 & 10 & 48.35 & 38.53 & 10 & 60.41 & 1.23 \\
$17-18$ & 16 & 16.81 & 16.45 & 15 & 26.63 & 16.52 & 5 & 52.00 & 8.58 \\
& & & & & & & & & \\
A $10-11$ & 17 & 26.32 & 20.09 & 9 & 38.00 & 14.14 & 11 & 42.27 & 22.66 \\
$15-16$ & 8 & 11.75 & 8.71 & 4 & 11.50 & 9.07 & 16 & 23.13 & 9.80 \\
$17-18$ & 6 & 14.33 & 8.96 & 4 & 13.00 & 5.87 & 16 & 23.00 & 7.66 \\
\hline
\end{tabular}

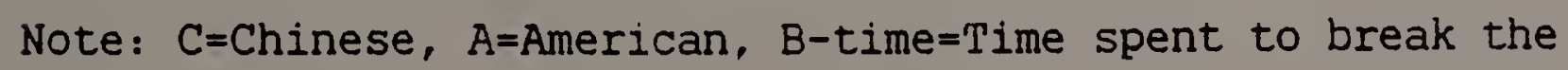
boundary, S-time=Time spent to solve the problem, G-time=Time spent before giving up, SD=Standard deviation. 
Table 4.7

The Average Trials Made by Each Age Group

to Break the Boundary, to Solve the Problem and before Giving Up

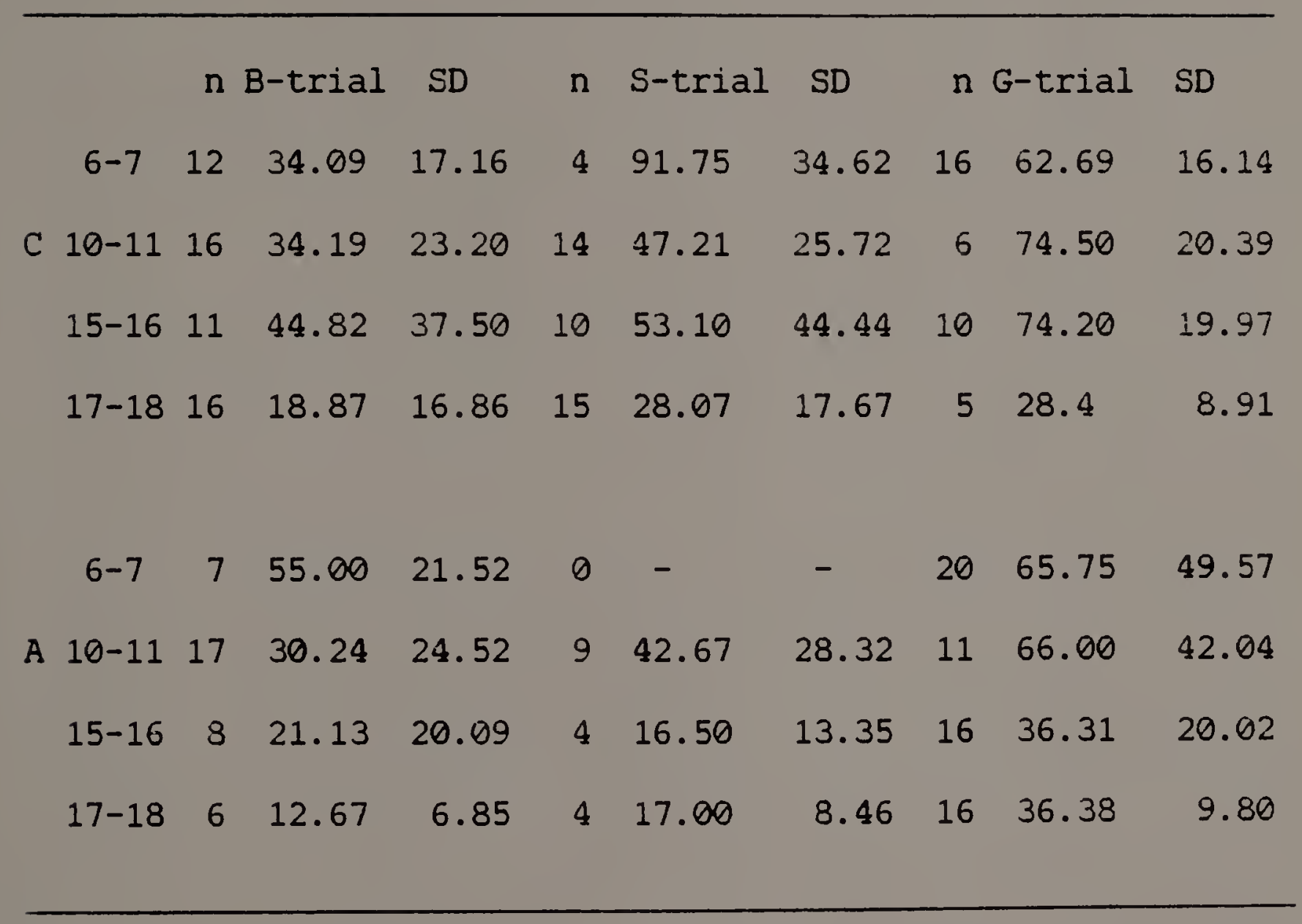

Note: $C=$ Chinese, $A=A m e r i c a n, B$-trial=Trials made to break the boundary, S-trial=Trials made to solve the problem, G-trial=Trials made before giving up, $S D=$ Standard deviation. 
Table 4.8

Summary of Univariate $\mathrm{F}$ Tests on Age Effect

\begin{tabular}{lcccc}
\hline Variable & Hypoth.DF & Error DF & $F$ & Sig. of F \\
B-time & 3 & 78 & 4.7985 & $.00404^{* *}$ \\
B-trial & 3 & 78 & 4.6301 & $.00494^{* *}$ \\
S-time & 3 & 44 & 4.8349 & $.00540^{*}$ \\
S-trial & 3 & 44 & 2.8629 & .04287 \\
G-time & 3 & 84 & 25.2182 & \\
G-trial & 3 & 84 & 4.9387 & $.00330^{* *}$ \\
\hline
\end{tabular}

Note: B-time=Time spent to break the boundary, B-trial= Trials made to break the boundary, S-time=Time spent to solve the problem, S-trial=Trials made to solve the problem, G-time=Time spent before giving up, G-trial=Trials made before giving up. ** means $p<.01$ after adjustment. 


\section{Hypothesis 3 Sex Effect}

The number of male and female boundary breakers $(20$ and 18 . respectively) and problem solvers ( 8 and 9 , respectively) in the American sample was very similar. Differences were also not great for male and female Chinese boundary breakers (30 and 25 , respectively) and problem solvers (24 and 19, respectively). No overall sex difference was found in the number of solvers or the number of boundary breakers $\left(x^{2}=0.43, d f=1, p>.05 ; X^{2}=1.26\right.$, $\mathrm{df}=1, \mathrm{p}>.05)$

No significant $\mathrm{F}$ value was obtained from the multivariate tests to show an effect of sex on any dependent variables (see Table 4.1, Table 4.2 and Table 4.3 ).

\section{Hypothesis 4 Interaction between Culture and Age}

A multivariate test revealed a significant interaction between culture and age in the time spent on the problem before giving up (see Table 4.3 ) but not in the time and trials to break the boundary (see Table 4.2) and solve the problem (see Table 4.1). The 10-11-year-old Chinese spent the longest time (130 min.) on the problem before giving up. In contrast, the average time for the rest of the Chinese was 57 minutes and the average time for the Americans was 30 minutes (see Table 4.6). A significant difference was found in time spent on the problem by the unsuccessful subjects betweem the 6-7 year old and 10-11 year old Chinese children $(p<.01)$ and between the 10-11 year old and 15-16 year old American children $(p<.01)$. Within each culture, the difference between 6-7 year old and 17-18 year old was 
significant $(p<.01)$. There was also a sinificant difference in number of trials, but not the time spent, to extend beyond the boundary between 15-16 year old and 17-18 year old Chinese students $(p<.01)$ and between $6-7$ year old and $10-11$ year old American children $(p<.05)$. The difference in the time spent to solve the problem between the 6-7 and 17-18 year old Chinese was significant $(p<.01)$. The trials made to solve the problem were also found significantly different between the chinese children 6-7 and 10-11 year old $(p<.01), 15-16$ and $17-18$ year old $(p<.05)$. No significant difference was found in the time spent and trials made to solve the problem among the four American age groups (see Table 4.9, p.51, and Table 4.10, p.52).

Among Chinese subjects, the higher the age level the greater the percentage of subjects drawing lines extending beyond the boundary and solving the problem (see Table 4.11). In both cultures, differences in performance were significant between subjects age 6-7 and 10-11. After age 10-11, the changes in performance were less dramatic and the differences among older age groups were not statistically significant $\left(X^{2}=5.91, d f=1\right.$, $p=.06$ for the comparison between $15-16$ and 17-18-year-old Americans; $\mathrm{X}^{2}=2.46, \mathrm{df}=2, \mathrm{p}>.20$ for the comparison among the 10-11, 15-16 and 17-18-year-old Chinese). 
Table 4.9

Summary of Univariate $F$ Tests on Interaction between Culture and Age

\begin{tabular}{lcccc}
\hline Variable & Hypoth.DF & Error DF & F & Sig. of F \\
B-time & 3 & 78 & .86892 & .46093 \\
B-trial & 3 & 78 & 3.02585 & .03446 \\
S-time & 2 & 44 & .62565 & .53961 \\
S-trial & 2 & 44 & 1.45095 & .24534 \\
& & & & \\
G-time & 3 & 84 & 14.7692 & $.007 * *$ \\
G-trial & 3 & 84 & 2.62843 & .05551 \\
\hline
\end{tabular}

Note: B-time=Time spent to break the boundary, B-trial=Trials made to break the boundary, S-time=Time spent to solve the problem, S-trial=Trials made to solve the problem, G-time=Time spent before giving up, G-trial=Trials made before giving up. ** means $p<.01$ after adjustment. 
Table 4.10

Summary of Contrasts between Means of Age Groups in G-time, G-trial, B-time, B-trial, S-time and S-trial

\begin{tabular}{|c|c|c|c|c|}
\hline Variable & Contrast & Estimate & Standard Error & t Value \\
\hline \multirow[t]{4}{*}{ G-time } & $M 1-M 2$ & 71.31 & 7.4446 & $9.5788^{\star *}$ \\
\hline & M1 - M4 & 6.69 & 7.9676 & .8397 \\
\hline & M5 - M6 & 9.66 & 5.8376 & 1.6548 \\
\hline & $M 6-M 7$ & 19.14 & 6.0910 & 3. $1423^{* \star}$ \\
\hline \multirow[t]{3}{*}{ G-trial } & $M 1-M 2$ & 11.81 & 8.2401 & 1.4332 \\
\hline & $M 1-M 4$ & 34.29 & 8.8191 & $3.8428^{\star \star}$ \\
\hline & M5 - M8 & 29.37 & 5.7734 & $5.0871^{\star \star}$ \\
\hline \multirow[t]{4}{*}{ B-time } & $M 1-M 2$ & 3.91 & 7.7976 & .5014 \\
\hline & M3 - M4 & 17.83 & 7.9975 & 2.2290 \\
\hline & M5 - M6 & 5.41 & 9.1169 & .5934 \\
\hline & $M 6-M 7$ & 14.57 & 8.7546 & 1.6642 \\
\hline \multirow[t]{5}{*}{ B-trial } & $M 2-M 3$ & 10.63 & 7.0861 & 1.5001 \\
\hline & $M 3-M 4$ & 25.95 & 7.1320 & $3.6394^{\star \star}$ \\
\hline & M5 - M6 & 24.76 & 8.3332 & $2.9712^{\star}$ \\
\hline & $M 6-M 7$ & 9.11 & 7.9557 & 1.1451 \\
\hline & $M 7-M 8$ & 8.46 & 10.0213 & .8442 \\
\hline \multirow[t]{4}{*}{ S-time } & $M 1-M 2$ & 17.92 & 15.6710 & 1.1435 \\
\hline & $M 1-M 4$ & 48.87 & 15.5542 & 3. $1419^{\star *}$ \\
\hline & $M 3-M 4$ & 21.72 & 8.7408 & 2.4849 \\
\hline & $M 6-M 7$ & 26.50 & 16.6102 & 1.5954 \\
\hline \multirow[t]{4}{*}{ s-trial } & $M 1-M 2$ & 44.54 & 12.1906 & $3.6520^{\star \star}$ \\
\hline & $M 2-M 3$ & 5.89 & 8.9207 & .6620 \\
\hline & $M 3-M 4$ & 25.03 & 8.7782 & $2.8514^{\star}$ \\
\hline & $M 6-M 7$ & 26.17 & 12.9212 & 2.0254 \\
\hline
\end{tabular}

Note: M1, M2, M3, M4 are means of age groups $6-7,10-11,15-16$ and 17-18 repectively for the Chinese sample. M5, M6, M7, and $M 8$ are means of age groups $6-7,10-11,15-16$ and 17-18 respectively for the American sample.

* shows $\mathrm{p}<.05$; ** shows $\mathrm{p}<.01$. 
Table 4.11

Percentage of the Boundary-Breakers

Who Solved the Problem

\begin{tabular}{ccc} 
& American & Chinese \\
$6-7$ & 0 & 33.3 \\
$10-11$ & 52.9 & 87.5 \\
$15-16$ & 50.0 & 90.9 \\
$17-18$ & 66.7 & 93.8 \\
\hline
\end{tabular}

Hypothesis 5 Interaction between Culture and Sex

A significant interaction was found between cultural and sex factors. This finding emerged because American girls spent less time than American boys on solving the problem (mean=19.0 min. for girls; mean=33.63 min. for boys) while Chinese girls spent more time than Chinese boys on solving the problem (mean=51.19 min. for girls; mean=41.45 min. for boys) (see Table 4.12 ). A multivariate test indicated that the interaction between culture and sex was significant $(p<.05)$ only in the time spent and trials made to solve the problem (see Table 4.1 ) but not in the time spent and trials made to break the boundary (see Table 4.2 ) or before giving up (see Table 4.3). A univarate $F$ test on the 
interaction between culture and sex indicated that the interaction in trials to break the boundary was significant ( $p<.05$ after adjustment) but the interactions in time spent to break the boundary and in trials to solve the problem were not significant $(.05<p<.10$ after adjustment) (see Table 4.13$)$.

Table 4.12

Average Time Spent by Each Sex Group to Solve the Problem

\begin{tabular}{lllll}
\hline & & & S-time & Sd. \\
Chinese & Male & 24 & 41.45 & 36.43 \\
& Female & 19 & 51.19 & 25.97 \\
& & & & \\
American & Male & 8 & 33.63 & 20.55 \\
& Female & 9 & 19.00 & 9.39 \\
\hline
\end{tabular}


Table 4.13

Summary of Univariate $\mathrm{F}$ Tests on Interaction

between Culture and Sex

\begin{tabular}{lcccc}
\hline Variable & Hypoth.DF & Error DF & F & Sig.of F \\
B-time & 1 & 78 & 3.8252 & .05407 \\
B-trial & 1 & 78 & 5.7161 & $.01922^{\star}$ \\
S-time & 1 & 44 & .68150 & .41353 \\
S-trial & 1 & 44 & 4.4385 & .04087 \\
& 1 & 84 & 1.9213 & .16938 \\
G-time & 1 & 84 & .01438 & .90484 \\
G-trial & 1 & & & \\
\hline
\end{tabular}

Note: $B$-time=Time spent to break the boundary, B-trial=

Trials made to break the boundary, S-time=Time spent to solve the problem, S-trial=Trials made to solve the problem, G-time=Time spent before giving up, G-trial=Trials made before giving up. * means $p<.05$ after adjustment. 


\section{Additional Findings}

The first 20 trials of all participants were analyzed to see if trial limit had the same effect on different cultural, age and sex groups in their performance on the nine-dot problem. The results of the analysis can be summarized as follows:

When the first 20 trials were examined, the total number of boundary breakers and problem solvers for each cultural group decreased drastically. Twenty-two Chinese and 15 American subjects extended beyond the boundary. Eleven Chinese and seven American subjects solved the problem. These cultural differences were not statistically significant $\left(x^{2}=1.0016, d f=1, p>.20\right.$ for boundary breakers; $x^{2}=1.7227, d f=1, .10<p<.20$ for problem solvers).

With 20 trials as a limit, age differences, however, were still salient in the number of boundary breakers $\left(X^{2}=11.6385\right.$, $d f=3, p<.05)$ and problem solvers $\left(x^{2}=18.2695, d f=3, p<.01\right)$. The differences between 6-7-year-old and 10-11-year-old were striking $12: 12$ in number of boundary breakers, $0: 4$ in number of problem solvers, see table $4.14, \mathrm{p} .57)$.

Age differences among the Chinese were significant in number of boundary-breakers $\left(\mathrm{X}^{2}=8.7743, \mathrm{df}=3, \mathrm{p}<.05\right)$ and number of problem-solvers $\left(\mathrm{X}^{2}=11.1956, \mathrm{df}=3, \mathrm{p}<.05\right)$. These age differences in number of boundary-breakers and problem-solvers were not significant $\left(\mathrm{X}^{2}=6.8103, \mathrm{df}=3, .10>\mathrm{p}>.05\right.$ after adjustment $)$ among the four age groups of the American sample. 
Within 20 trials, 23 males and 14 females extended the boundary formed by the nine dots. Twelve males and six females solved the problem. It seems that more males extended the boundary and solved the problem than females. However, the overall sex differences were not statistically significant $\left(\mathrm{X}^{2}=\right.$ $\left.2.8477, d f=1, .10>p>.05 ; \quad X^{2}=2.2535, d f=1, p>.10\right)$

Table 4.14

Number of Boundary-Breakers, Problem-Solvers and Give-Ups within Twenty Trials

Chinese

American

B-Breaker P-Solver Give-Up B-Breaker P-Solver Give-Up $\begin{array}{lllllllllllll}M & F & M & F & M & F & M & F & M & F & M & F\end{array}$ $\begin{array}{ccccccccccccc}6-7 & 1 & 1 & 0 & 0 & 0 & 0 & 0 & 0 & 0 & 0 & 0 & 0\end{array}$ $\begin{array}{ccccccccccccc}10-11 & 4 & 2 & 1 & 1 & 0 & 0 & 2 & 4 & 1 & 1 & 0 & 1\end{array}$ $\begin{array}{rllllllllllll}15-16 & 4 & 0 & 2 & 0 & 0 & 0 & 3 & 2 & 1 & 1 & 2 & 1\end{array}$

$\begin{array}{rllllllllllll}17-18 & 8 & 2 & 6 & 1 & 2 & 0 & 1 & 3 & 1 & 2 & 1 & 0\end{array}$

$\begin{array}{lllllllllllll}\text { Total } & 17 & 5 & 9 & 2 & 2 & 0 & 6 & 9 & 3 & 4 & 3 & 2\end{array}$

Note: B-Breakers=Boundary-Breakers; P-Solvers=Problem-Solvers; Give-Up=subjects who gave up; M=Male and $F=F e m a l e$. 
No sex differences were found among 6-7 year old Chinese (see Table 4.14). From age 10-11, sex differences appeared. The older the age, the greater the differences. At age 17-18, the male to female boundary-breakers became $8: 2$. Male to female problem solvers became $6: 1$. These sex differences were significant $\left(\mathrm{X}^{2}=\right.$ $9.0282, d f=1, p<.01$ for boundary-breakers; $X^{2}=5.1647, d f=1, p<.05$ for problem-solvers).

For the American sample, the number of problem-solvers of the two sex groups was nearly the same in each age group. The numbers of boundary-breakers of male and female groups were not significantly different $\left(x^{2}=0.7385, d f=1 p>.20\right)$.

The relationship between constructing the shape of the solution within the boundary and solving the nine-dot problem was also explored. A common early attempt is to draw a triangular shape within the boundary (see Figure 4.2). Fifty-two percent of the Chinese and $22 \%$ of the American participants produced the triangle shape within the boundary on their first drawings and then later extended their lines beyond the boundary and solved the problem. Twenty-two percent of the 43 Chinese and $65 \%$ of the 17 Americans who solved the nine-dot problem produced the triangle shape within the boundary in their early trials.

For the Chinese group, the percentage of people who drew the triangle shape of the solution within the boundary decreased to zero as the age increased to 17-18. For the American group, no such tendency was found (see Table 4.15). 


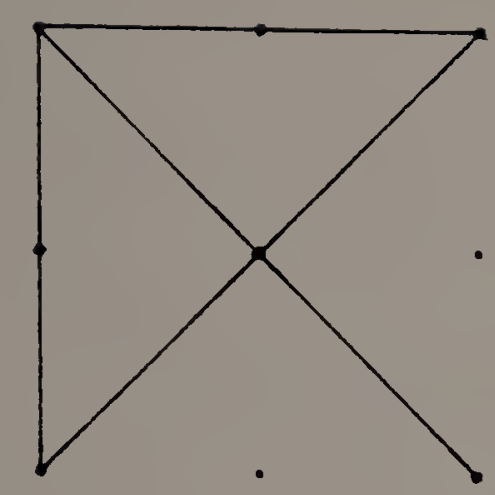

Figure 4.2

Triangle-Shape Solution within the Boundary

Table 4.15

Percentage of Students Who Drew the Shape of the Solution within the Boundary

\begin{tabular}{ccc} 
& American & Chinese \\
$6-7$ & 85 & 25 \\
$10-11$ & 70 & 65 \\
$15-16$ & 35 & 25 \\
$17-18$ & 65 & 0 \\
\hline
\end{tabular}


A new version of the solution to the nine-dot problem was created coincidentally by 40 Chinese and 3 American participants This version is an open rather than closed triangle shape. It follows the direction more closely than th old version (see Figure 4.3 ) in terms of not retracing or retouching the dots by not starting from the comer dot. In the old version, the first line begins on a corner dot and, the intersection between the third and fourth lines is also on the corner dot. Thus, the corner dot is retouched.

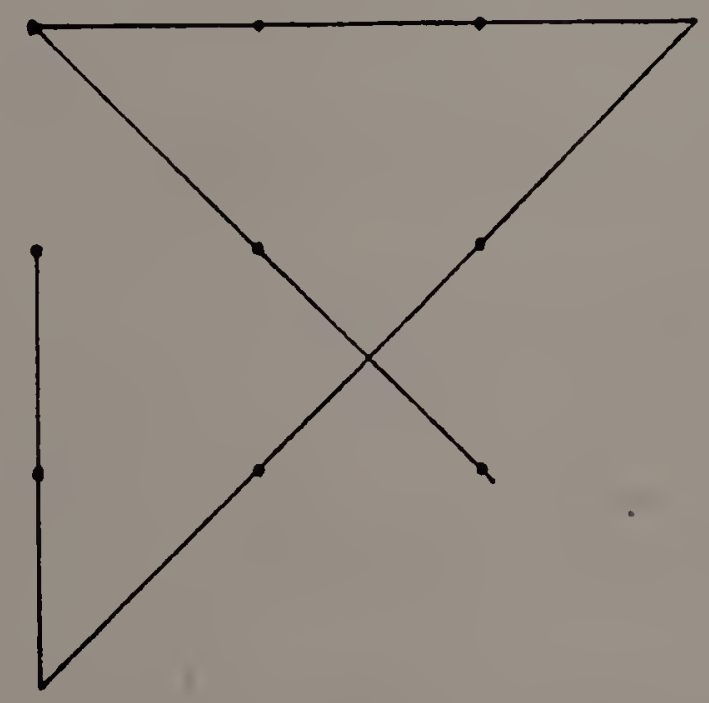

Figure 4.3

The New Version of the Solution 
But it might be controversial because the dot on the top of the triangle is only linked to the dot on one side while not linked to the dot on the other side. An argument for this new solution may be that it has linked the nine dots with four straight lines without retracing and without lifting the pen from the paper. Since it is not stated in the requirement of the task that any dot should be connected to its neighbors directly this new version of solution to the nine-dot problem is legal. Ninety-three percent of the Chinese solvers presented this new version as their solution, and $23 \%$ of them rejected the old version in their trials before they reached the new version. Meanwhile, $18 \%$ of the American solvers presented the new version as their solution. None of them rejected the old version as their Chinese counterparts did. 
CHAPTER 5

DISCUSSION

\section{Hypothesis 1 Culture Effect}

The results of this study revealed cultural differences in performance on the nine-dot problem. Fewer Americans went beyond the boundary formed by the dots and solved the problem compared to the Chinese participants.

One factor that may have contributed to this cultural difference is the different approach to the task taken by the two cultural groups. Most American participants spent more time on trials to obtain the solution (This may reflect their value of doing.) while most of the Chinese participants spent more time on figuring out what was required of them (This may reflect their value of "thinking three times before doing".). The Chinese followed the direction so strictly that $93 \%$ of the solvers produced a solution which avoided touching a dot twice (see Figure 4.3 ) and $23 \%$ of them even rejected the old version of the solution in their trials.

The directions for the nine-dot problem are very general. The directions do not specify a) whether the lines may be extended beyond the boundary formed by the dots, b) whether one dot may be connected to two or three lines, c) whether the lines may cross each other, or d) whether the lines may intersect at locations other than a dot. Thus the problem solvers are free to make 
assumptions about all of these conditions. By referring to the direction now and then in the problem-solving process, especially when having failed to reach a solution after exploring various trials under certain assumptions, one may have more chance to challenge the assumptions and be aware of what is required and what is not. Therefore, one is more likely to break the assumed boundary and solve the problem. In this sense, clarifying the requirement may have helped the Chinese participants in solving the problem.

Another factor which may account for why there were more solvers from the Chinese group is that the Chinese participants on average spent a longer time on the problem and completed far more trials in their efforts to arrive at a satisfactory solution. In contrast, the American participants on average spent a shorter time on the problem. Their pace was faster than their Chinese counterparts.

Chinese students may have more training and exercises in skills for solving mathematic problems than American children. This training may provide the students with knowledge of straight lines, shapes and spatial relationships as well as strategies for representing and attacking the problem and monitoring the problem solving process.

\section{Hypothesis 2 Age Effect}

Significant age differences were found in the number of solvers, the time spent on the problem, the time taken to break the boundary, and the time taken to solve the problem. The change 
in performance on all of these measures was most striking and dramatic between age 6-7 to age 10-11. After age 10-11, changes in performance were minimal. These facts are consistent with Piaget's (1970) stages of intellectual development. The four age groups $(6-7,10-11,15-16,17-18)$ correspond to four cognitive developmental stages -- late preoperational, late concrete operational, formal operational and late formal operational.

Cognitive ability develops from a lower level to a higher level. The higher the stage, the more one is cognitively ready to solve complex problems. Development is based on maturation and experience, whereas maturation and experience normally grow with age. According to Piaget (1970), in the preoperational stage (age 2-7), language develops and thought becomes a symbolic process, but thinking is still perception bound. By the age of 6-7, children may understand the words in the instructions for the nine-dot problem but not the abstract concepts involved. Their egocentrism, centration and inability to perform an operation requiring reversibility may prevent them from solving the problem. In the concrete operational stage (7-11), thought becomes reversible, conservation becomes operative and ability to solve concrete problems develops. Logical operations develop although thinking is experience-based. Given the visual and hands-on nature of the nine-dot problem along with its simple instructions, it is possible for a 10-11-year-old to solve the problem. The jump from $6-7$ to $10-11$ in number of solvers may reflect a qualitative change of cognitive ability. 
In the formal operational stage (12-adulthood), formulation and testing hypotheses, abstract thought, deductive reasoning and hypothetico-deductive reasoning develop. Thought is no longer perception bound. At this stage, one is cognitively and developmentally ready for solving complex problems. The fact that the partcipants age 15-18 required less time to solve the problem than the younger childern (see Table 4.6) may reflect the progress of cognitive development.

As reported in Chapter 4 , age differences in the number of boundary breakers were not significant for the Chinese group. If the data from age 10-11 group were excluded, the age difference in the number of boundary breakers would not be significant for the American group, either. Age effects do not appear in the product of boundary breaking but in the solution. Since the number of boundary breakers of the age 6-7 group is not different from that of the older groups $(15-16,17-18)$ who are assumed to be in the formal operational stage, it may be inferred that breaking the boundary does not necessarily require formal operations but solving the problem does.

\section{Hypothesis 3 Sex Effect}

Although sex differences were found in previous studies (Maier \& Casselman, 1970; Lung \& Dominowski, 1985), this study did not find an overall sex difference in the number of boundary breakers or solvers of the problem or any other dependent variables. One interpretation of this discrepancy in findings may be that the previous studies placed time limits on subjects while 
the current study allowed participants to work at their own pace. And the previous studies were conducted within one culture while the current study was conducted in two cultures. The interactions between culture and sex -- the Chinese girls did spend more time than Chinese boys on solving the problem whereas American girls spent less time than American boys on solving the problem -- may have covered the overall sex differences statistically.

Hypothesis 4 Interaction between CuIture and Age

The interactions between cultural and age factors manifested themselves in two ways: First, the $10-11$ year old Chinese spent twice as long as the rest of the chinese groups and three times as long as their American counterparts on the problem before giving up. This fact may indicate that this group had greater motivation and persistence in solving the problem. To answer why this is so, further studies are needed. However, some potential explanations may be offered. First of all, the 10-11 age group was old enough to appreciate this challenging and interesting problem. At this age, heavy school work had not severely disrupted their curiosity yet. In the second place, their education taught them that there must be a solution to this kind of problem and they were supposed to find it. In the third place, they were brought up in a culture that values achievement, persistence and hard work. They seldom hear their parents or other adults say to them "Have fun" or "enjoy yourself" which many of their American counterparts hear frequently. 
Second, no one in the 17-18 year old Chinese group drew the triangle solution shape within the boundary which was frequently seen in all other groups. One explanation for this phenomenon may be that participants in this group were able to predict that the triangle solution within the boundary could not solve the problem according to the requirements. The fact that they needed fewer trials to solve the problem showed their thoughtfulness and better self-monitoring than other groups. Among the eight groups, this group had the highest number of solvers and the highest percentage of boundary breakers who solved the problem. Among the four Chinese groups, the 17-18 year old group took the shortest time and number of trials to break the boundary and to solve the problem. Highly developed logical thinking and self-monitoring strategies in problem solving may have contributed to their success.

\section{Hypothesis 5 Interaction between Culture and Sex}

As reported in Chapter 4 , the interaction between culture and sex was displayed only in the time spent and trials attempted to solve the problem. The American girls were faster than the boys while the Chinese boys were faster than the girls. This interation between culture and sex is coincident with the findings of interactions between culture and sex (Chang, 1984 ) in cognitive learning styles (field dependence and field independence). Acculturation may contribute to an individual's cognitive style as well as the solving of the nine-dot problem. To explain the relationship between cognitive style and the 
performance of the nine-dot problem solving, further studies are needed. Meanwhile, it is important to note that when allowed to work on the problem at their own pace, the Chinese girls could solve the problem as well as boys, and the American boys could do as well as girls. However, due to the speed difference, the Chinese girls may not be able to solve the problem if forced to stop within a shorter time limit.

\section{Additional Findings}

Within the 20 trial limit, the cultural differences in number of boundary breakers and problem-solvers became insignificant, but age differences and interactions between culture and age and culture and sex were significant (see Additional Findings, Chapter 4). Age and sex differences were salient among the Chinese but not among the Americans. The older Chinese particicants were more likely to go beyond the boundary and solve the problem than younger ones. Boys were more likely to go beyond the boundary and solve the problem than girls. If we consider these findings together with the results reported in hypotheses 1-5 of Chapter 4, we can see that the twenty-trial limit did not affect the productivity of the American group as much as it affected the Chinese group. Within the Chinese group girls were more affected by the trial limit than boys and younger children were more affected than older children.

Lung and Dominowski (1985) expected that if the subjects learned the proper representation and strategy, they should be able to apply these to the nine-dot problem and overcome the 
difficulty caused by the shape of the pattern. One accidental finding of this study provides support for Lung and Dominowsiki's expectation. The 6-7 year old Chinese pupils could solve the nine-dot problem after having courses of thinking skills whereas their peers without such training could not solve the problem (refer to Subjects in Chapter 3 ). This finding suggests that training on thinking skills can facilitate the solving of difficult problems such as the nine-dot problem for even young children. Another accidental finding was that the 10-11 year old American pupils who did well on the nine-dot problem had been taught by one teacher who emphasized problem solving strategies in math class. Strategies needed in the nine-dot problem solving may be taught through math or other subjects.

The Nature of the Difficulty of the Nine-dot Problem

As discussed in Chapter 2, there are different perspectives on the nature of the difficulty of the nine-dot problem. The representative ones are the perceptual-organization view and the strategy view. The current study has provided evidence to support both views.

According to the perceptual-organization view, the very way the problem is presented elicits perceptual responses that retard problem solution. The vague directions of the problem leave solvers to make assumptions about the task. One evidence for this view is that almost all the participants across cultures and age made their first trials within the square space formed by the dots. Some eventually went out of the boundary but others never 
did. Another piece of evidence is the new version of solution to the nine-dot problem developed by $93 \%$ of the Chinese solvers. Twenty three of the new solution producers had rejected the old version in their trials. The new version avoided touching a dot more than once. This new solution may reflect the solvers' assumption about a condition -- a dot should not be touched more than once. Fortunately, these solvers did not make another restrictive assumption simultaneously. If they had assumed that each dot should be connected to its neighbors directly, they would not have been able to solve the problem. The difference in the number of solvers between the two cultural groups may indicate that the extent the assumed boundaries affect the nine-dot problem solving varies from culture to culture.

The current study can also offer empirical data (see Chapter 4) to modify, from a cognitive developmental perspective, the statement that the solution to the nine-dot problem lies in going beyond what appears to be the boundary. This statement is true for people who are cognitively operating at a formal operational stage or have had training in thinking skills and/or problem solving strategies, but this statement is not true for people who are operating below formal operational level and without training in thinking skills or problem solving strategies.

The very fact that $100 \%$ of 6-7 year old Americans and $67 \%$ of 6-7 year old Chinese who went beyond the boundary formed by the dots but failed to solve the problem supports the strategy view. According to the strategy view, whether one has the knowledge of 
the field and the strategies to attack the problem and to monitor one's own steps are crucial for solving the problem. It is difficult because the solvers do not have the required strategies in their repertoire. The mere behavior of going beyond the boundary may be the result of trial-and-error, or may involve certain problem solving strategies such as representing the problem appropriately. Apparently, those 6-7 year olds do not have all the required strategies to solve the problem.

\section{Limitations}

One of the difficulties in cross-cultural research is determining how to obtain matched samples. It is very hard to find equivalent groups in two cultural settings. Although the age, sex and type of school children attended were generally matched, the setting of the test was quite different. In China, the randomly chosen participants were asked to do the task in a quiet classroom after school. In the United States, the participants had to do the task during school hours. Since the number of students in each class or grade is smaller in Massachusetts than in Beijing, the pool for sampling is smaller, too. As a result, half of the 10-11 year old American sample were taught by the same teacher so that another variable was introduced (This factor was considered earlier when discussing the data.).

The sample from Beijing did not represent people from rural areas nor national minorities so that the findings from this study can not be generalized to those populations. The sample 
from Massachusetts did not represent people from private schools, big cities nor very rural areas so that findings from this study can not be generalized to those populations.

\section{Recommendations for Further Research}

To test the effect of the assumed boundaries on the nine-dot problem solving of bilingual and bicultural populations, additional data from Chinese Americans have been collected and will be analyzed to shed light on a number of issues raised in this study. Meanwhile, packages of boundary breaking games and exercises are being developed for schools to facilitate creative problem solving.

The results of the current study supported the author's hypotheses of cultural and developmental differences in nine-dot problem solving. At this moment, we do not know what the results would be if populations from rural areas, big cities and private schools were included in our sample. To gain more representative data of the two cultures, we need to study these populations as the next step.

To test the research hypotheses further, i.e., the universality of the effect of assumed boundaries on problem solving in different ethnic groups, we need to collect data from more cultures.

To explore the effect of the assumed boundaries on problem solving further, we also need to include other insight problems in our study. 


\section{APPENDIX A}

WORKSHEET

\section{Directions}

Welcome and thank you for joining us in creative problem solving. Your task is to connect all the nine dots by drawing four straight lines. Go through each dot only once. Do not lift your pencil from the paper.

Please try your hand on the nine dots printed on the worksheets. Now start from 1 , and then try on $2,3,4, \ldots$ until you find the solution. When you use up all the worksheets, you may ask the teacher for more.

Remember, all your trials are important, whether they are right or wrong. Please do not erase any solution trials. Just move to the next nine dots and make another trial. The teacher is recording the time you take on the task. But it is $0 . k$. to have many, many trials and spend a long time on the task. As long as you try your best, you are contributing to our creative problem solving.

Enjoy it. 
Worksheet

1

Turn to next page. 


\section{Worksheet}

7

Turn to next page. 
Worksheet

19

21

Turn to next page. 
Turn to next page.
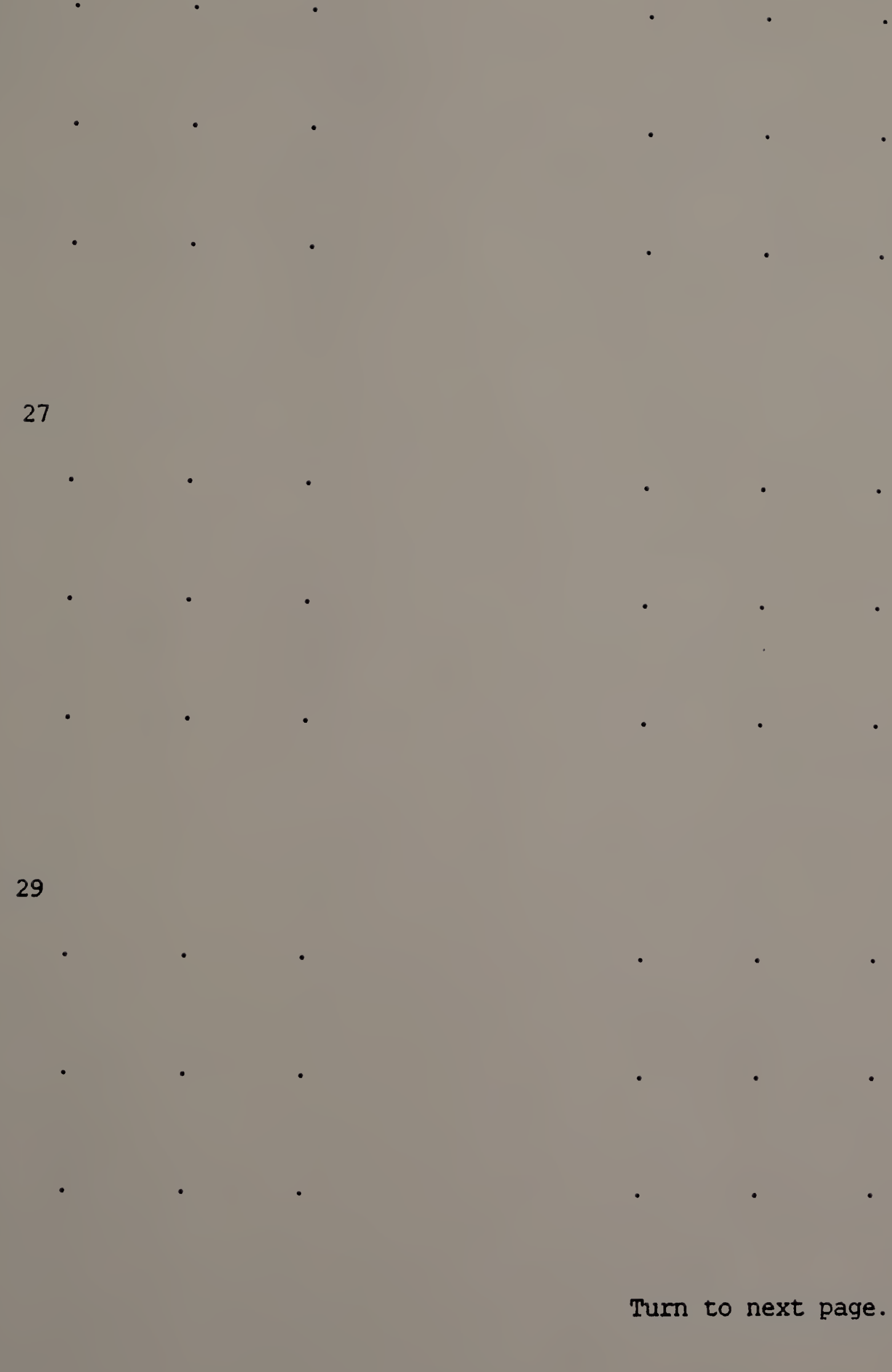

(1)

27

8
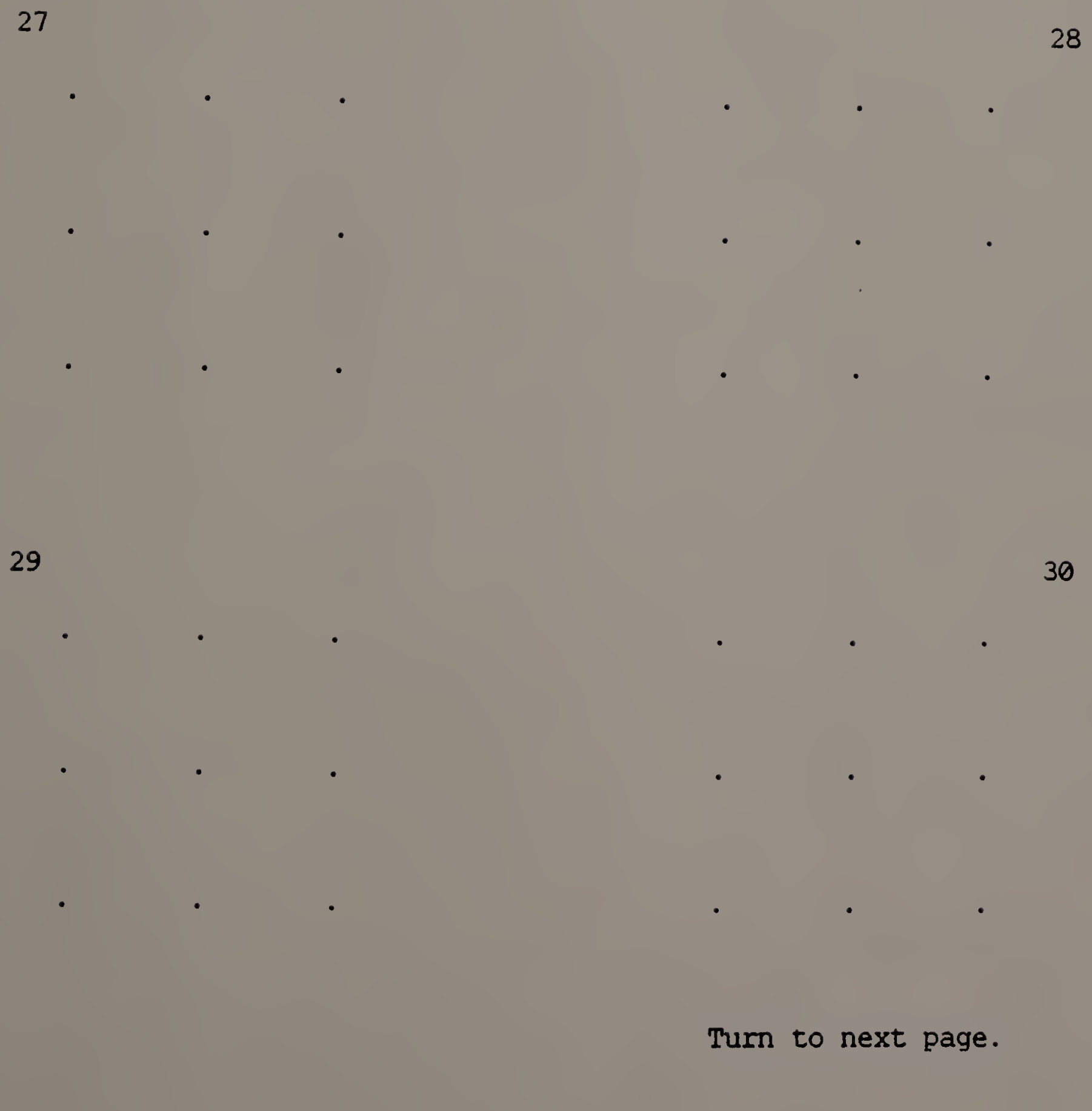

30

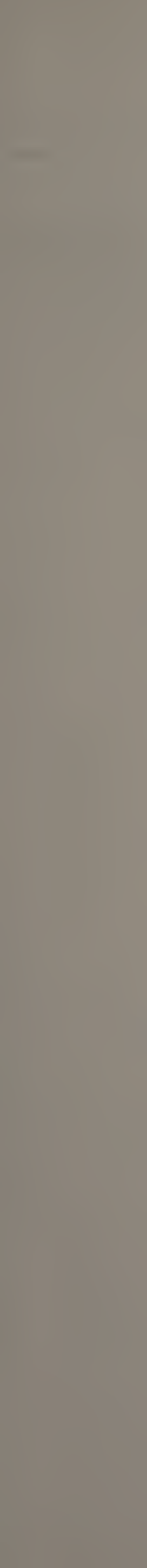

(n)

29

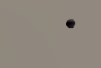

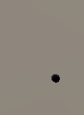

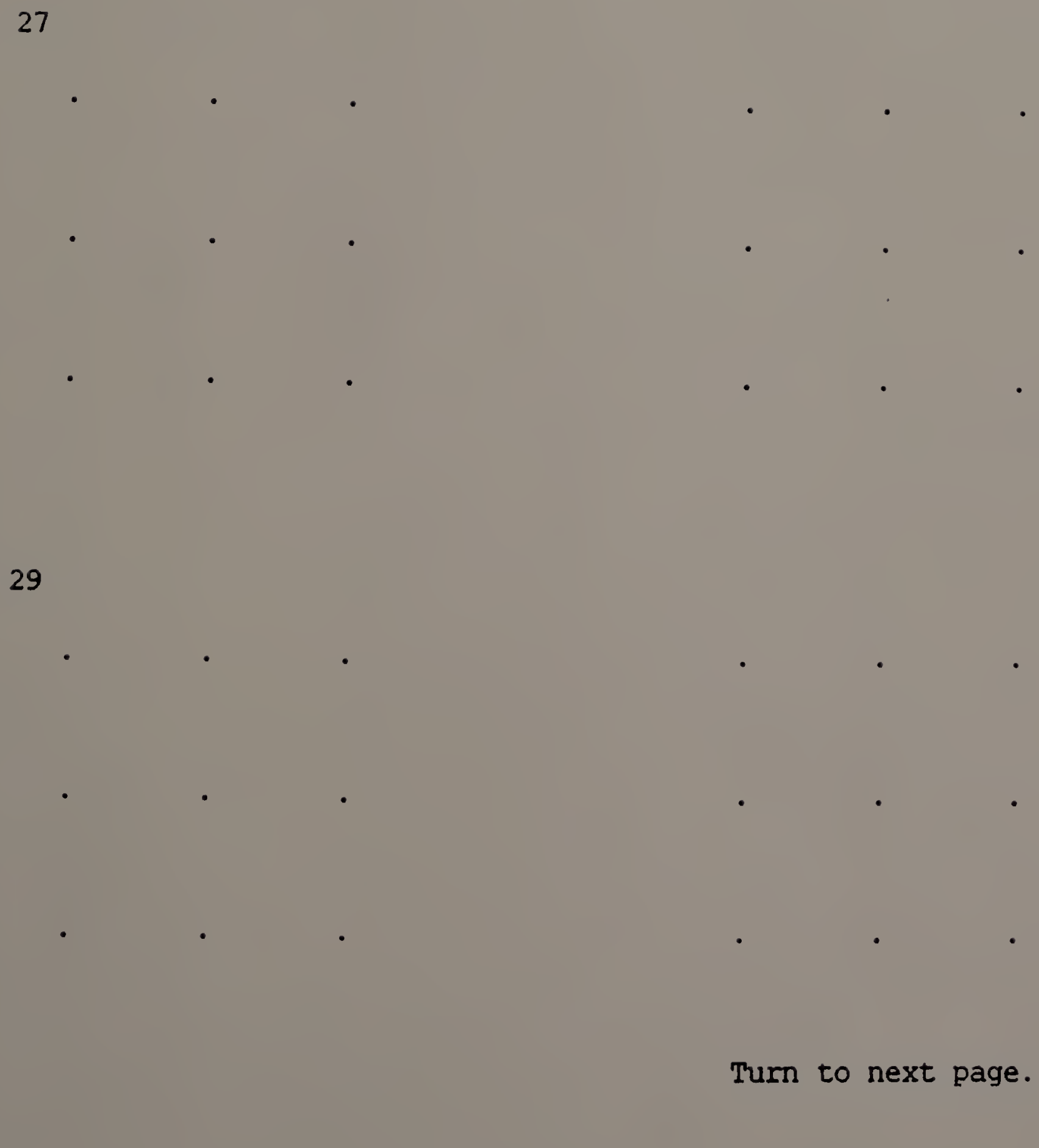
Then to next page.
$+$

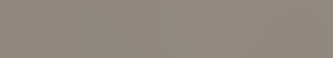

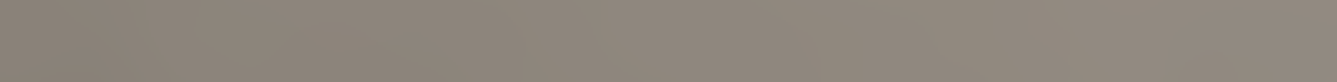

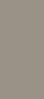

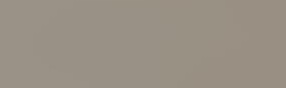
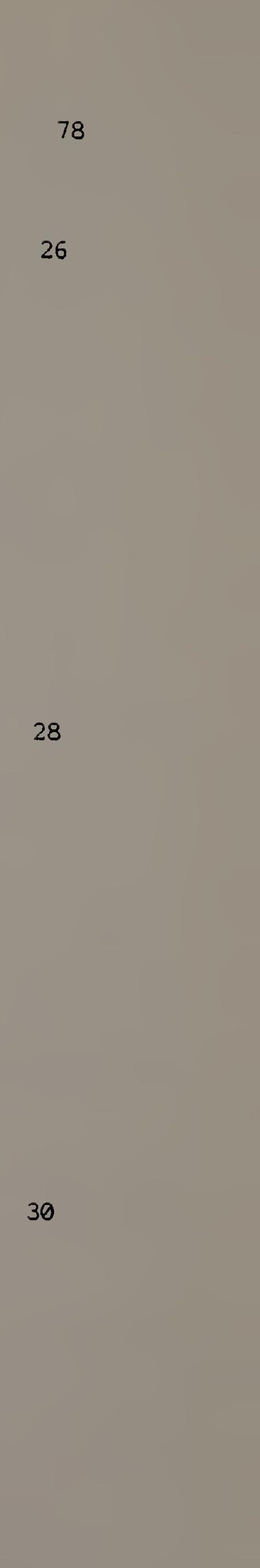

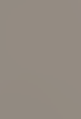

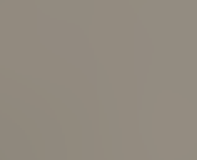

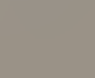

30

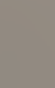
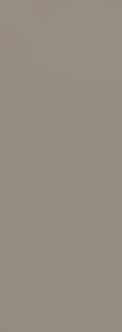
79

Worksheet 


\section{APPENDIX B}

\section{TIME RECORDING SHEET}

\section{Date:}

No:

Age:

Grade:

Sex:

Time started:

Time extended the boundary:

Time solved the problem:

Time gave up:

Note: Time recording must be accurate to the level of minute. 


\section{APPENDIX C \\ GRAPHS OF NINE-DOT PROBLEM AND ELEVEN-DOT PROBLEM}

The Graph of Nine-Dot Problem:

The Graphic Solution:

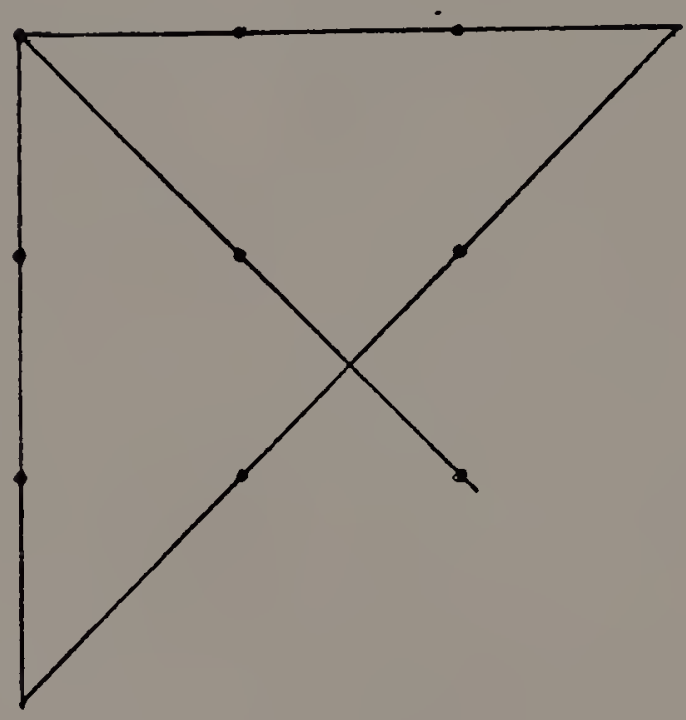

The Graph of Eleven-Dot Problem:

The Graphic Solution:

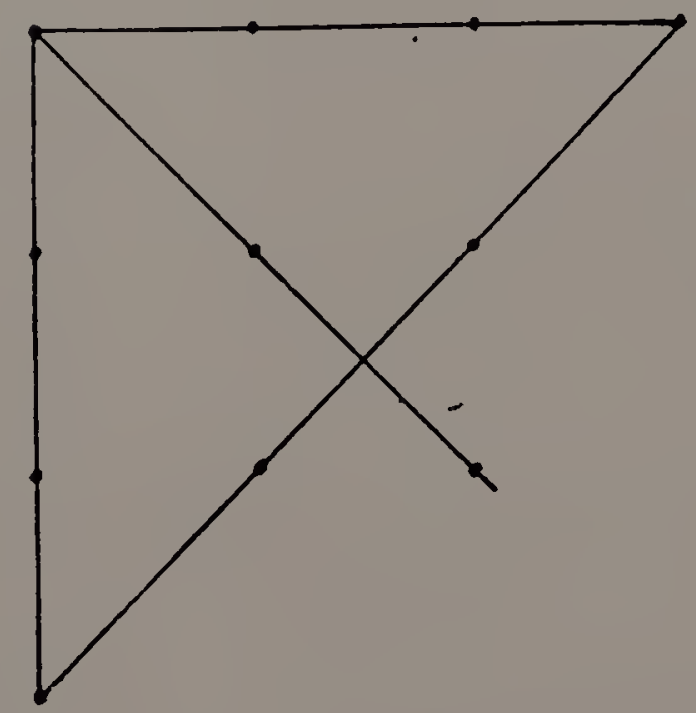




\section{REFERENCES}

Berthelot, R. J. (1982). Learning styles of females and implications for vocational-technical education: A review of the literature. Final Report from July 1, 1981 to June 30 , 1982. Pensacola Junior College, Florida. Florida State Dept. of Education, Tallahassee. Div. of Vocational Education. 19 p.

Burnham, C. A., \& Davis, K. G. (1969). The nine-dot problem: Beyond perceptual organization. Psychonomic Science, 17 , 321-323.

Chadha, N. K. (1985). Creativity and cognitive style. Psycho Linqua, $15(2), 81-88$.

Chang, W. C. (1984, August). An experimental study of selfconcept and cognitive style as indicators of psychological differentiation. Paper presented at the International Conference on Thinking (Cambridge, MA, August 19-23, 1984). 29 p.

Chatterjea, R. G., \& Paul, B. (1982). Cognitive style, social environment, sex and recognition capacity. Psycho Linqua, 12 (1), 37-45.

De Bono, E. (1973). Lateral thinking--Creativity step by step. New York: Hanger Colophon Books, Harper \& Row.

Dominowski, R. L. (1981). Comment on " An exanimation of the alleged role of 'fixation' in the solution of several 'insight' problems by Weisberg and Alba. Journal of Experimental Psychology: General, 110 (2), 199-203.

Duncker, K. (1945). On problem solving. Psychological Monograghs, $\underline{58}(5)$.

Fowler, C. J., \& Fowler, J. F. (1984). The consistency of Nigerians performance on three tasks measuring field dependence. International Journal of Psychology, 19 (3), 271-278.

Gauci, E. (1983, August). Applying empirical methods in comparative education: An example. In: Comparative and international studies and the theory and practice of education. Proceedings of the Annual Conference of the Australian Comparative and International Education Society (11th, Hamilton, New Zealand, August 21-24).

Glass, A. L., Holyoak, K. J., \& Santa, J. L. (1979). Cognition. Reading, MA: Addison-Welsley. 
Greeno, J. G. (1978). Natures of problem solving abilities. In W. K. Estes (Ed.), Handbook of Learning and Cognitive Process (Vol.5). Hillsdale, NJ : Erlbaum.

Hall, E. T. (1966a). The hidden dimension. Garden City,N.Y.: Doubleday.

Hall, E. T. (1966b). The silent lanquage. Garden City,N.Y.: Doubleday.

Hernandez, J. E. (1986). A cross cutural longitudinal study of the relationship between mother's cultural values and creativity in children and adolescents. Dissertation Abstracts International, Vol.46 (10-B), 3639.

Kendler, M. (1963). Basic psychology. New York: Meredith Publishing Company.

Khatena, J., \& Zetenyi, T. (1983). Production of non-sound images given sound stimuli in a cross-cultural setting. Perceptual and Motor Skills, 56 (2), 505-506.

Krech, D., \& Crutchfield, R. S. (1968). Elements of psychology. NY: Alfred A. Knopf

Levine, M. (1975). A cognitive theory of learning. Hillsdale, NJ: Erlbaum.

Lin, P. C. (1982). The relationship of field independence to concrete operations and formal operations. Acta Psychologica Taiwanica, 24(2), 101-111. In Chinese.

Loader, E. C. , Edwards, S. W. , \& Henschen, K. P. (1982). Field-dependent/field-independent characteristics of male and female basketball players. Perceptual and Motor Skills, 55 (3, pt 1), 883-890.

Lung, C. T., \& Dominowski, R. L. (1985). Effects of strategy instructions and practice on nine-dot problem solving. Journal of Experimental Psychology: Learning, Memory, and Cognition, 11 $(4), 804-811$.

Luchins, A. S. (1942). Mechanization in problem-solving. Psychological Monographs, 54(6).

Lundsteen, S. W. (1980). Measurement for creative problem solving in kindergarten children. Paper presented at the national council on measurement in education, April 8, Boston, MA. 
Maier, N. R. F. (1930). Reasoning in humans: I. On direction. Journal of Comparative Psychology, 10, 115-143.

Maier, N. R. F. (1940). The behavior mechanisms concerned with problem solving. Psychological Review, 47, 43-53.

Maier, N. R. F., \& Casselman, G. G. (1970). Locating the difficulty in insight problems: Individual and sex differences. Psychological Reports, 26, 103-117.

Mwamwenda, T. S., Dionne, J. P., \& Mwamwenda, B. B. (1985). Theoretical and empirical link between psychological differentiation and extraversion. Psychological Reports, 56(1). $147-154$.

Newell, A., \& Simon, H. (1972). Human problem solving. Englewood Cliffs, NJ: Prentice-Hall.

Pandey, A. K., \& Pandey, A. K. (1985). A study on cognitive styles of urban and rural college students. Perspectives in Psychological Researches, $\underline{8}(2), 38-43$.

Plaget, J. (1970). Science and education and the psychology of the child. New York: Orion.

Rabinsky, L. B., \& Kumar, V. K. (1979). A cross-cultural study in creativity, Isreali and American students, grade 9 through 12 . Paper presented at the Annual Meeting of the American Education Research Association, held in April, San Francisco, CA.

Rimm, S., \& Davis, G. A. (1980). Five years of international research and GIFL: An instrument for the identification of creativity. Journal of Creative Behavior, 14(1), 35-46.

Sapir, E. (1929). The status of linguistics as a science. Lanquage 5, 207-214.

Scheerer, M. (1963). Problem-solving. Scientific American, 208, 118-128.

Shallcross, D. (1981). Teaching creative behavior, How to evoke creativity in children of all ages. Englewood Cliffs, NJ: Prentice Hall.

Shepard, J. M. (1981). Sociology, St. Paul, New York: West Publishing Company.

Simon, H. A. (1978). Information processing theory of human probem solving. In W.K. Estes (Ed.), Handbook of Learning and Cognitive Processes, $\underline{5}$, Hillsdale, NJ: Erlbaum. 
Swinnen, S. (1984). Field dependence independence as a factor in learning complex motor skills and underlying sex defferences. International Journal of Sport Psychology, 15(4), 236-249.

Torrance, E. P., \& Sato, S. (1979). Figural creative thinking abilities of United States and Japanese majors in education, Creative Child and Adult Quarterly, $\underline{4}(4), 216-221$.

Weisberg, R. W., \& Alba, J. W. (1981a). An examination of the alleged role of "fixation" in the solution of several "insight" problems. Journal of Experimental Psychology: General, 110(2), 169-192.

Weisberg, R. W. \& A Alba, J. W. (1981b). Gestalt psychology, insight, and past experience: Reply to Dominowski. Journal of Experimental Psychology: General, 110(2), 193-198.

Weisberg, R. W., \& Suls, J. M. (1973). An information-processing model of Duncker's candle problem. Cognitive Psychology, $\underline{4}$, 255-276.

Whorf, B. L. (1956). Lanquage, thought and reality. Ed. by J. E. Carroll. Cambridge, M.A.: M.I.T. Press. 
